\title{
THE SOLUTIONS OF THE MATHIEU EQUATION WITH A COMPLEX VARIABLE AND AT LEAST ONE PARAMETER LARGE*
}

BY

\author{
RUDOLPH E. LANGER
}

Introduction. The Mathieu differential equation

$$
\frac{d^{2} u}{d z^{2}}+\{\Delta-\Omega \cos 2 z\} u=0
$$

also commonly known as the equation of the elliptic cylinder functions, is too well known to require any introduction. Its solutions govern problems of the greatest diversity in astronomy and theoretical physics, and have accordingly been the subjects of a vast number of investigations. $\dagger$

The differential equation as such depends upon two independent parameters, designated in the form written above by $\Delta$ and $\Omega$. In the present discussion these are to be taken real but are to be numerically unrestricted except that at least one is to be large. The variable will be permitted to range over the complex plane.

Since the coefficient of the differential equation is an even simply periodic analytic function of $z$, it is known from Floquet's theory of such equations that the solutions are in general of the structure

$$
u(z)=c_{1} e^{\mu z} \phi(z)+c_{2} e^{-\mu z} \phi(-z),
$$

in which the function $\phi(z)$ is periodic. The characteristic exponent, $\mu$, is a constant as to $z$ but depends in an intricate way upon the parameters $\Delta$ and $\Omega$. If it is real, the equation obviously possesses a solution which for large real values of the variable becomes exponentially infinite, i.e., a so called unstable solution. In the alternative case the exponent is pure imaginary and the solutions remain bounded along the axis of reals, i.e., are of the so called stable type. The intermediate case in which $\mu=0$ is of especial im-

* Presented to the Society, April 6, 1934; received by the editors February 12, 1934.

$\dagger$ Cf. for the hiterature and for partial enumerations of applications of the equation: Strutt, M. $j$. O., Lamesche-Mathieusche und verwandie Funktionen in Physik und Technik, Ergebnisse der Mathematik und ihrer Grenzgebiete, vol. 1, No. 3, Berlin, 1932; Whittaker and Watson, A Course in Modern Analysis, 3d edition, 1920, Cambridge University Press; Humbert, P., Fonctions de Lamé et Fonctions de Mathieu, Mémorial des Seiences Máthematiques, X, Paris, 1926; Van der Pol, B., and Strutt, M. J. O., On the stability of the solutions of Mathieu's equation, The Philosophical Magazine, vol. 5 (1928), p. 18. 
portance, for the equation then admits one solution known as a Mathieu function which is periodic. The second solution, a Mathieu function of the second kind, is then not periodic and is of a functional structure distinct from that indicated above.

With either of the parameters $\Delta$ and $\Omega$ fixed, the relation $\mu=0$ restricts the remaining one to a denumerably infinite set of values called the characteristic values. Broadly speaking the determination of these values and of the corresponding Mathieu functions is the matter of prime importance in the applications of the equation which belong more immediately to the domain of physics, while the determination of the characteristic exponent in terms of a fixed set of parameters is generally the peculiar requirement of the applications to astronomy.

When the values of the parameters are small the solution of the differential equation is generally and appropriately essayed through the means of convergent series expansions. When at least one of the parameters is large, on the other hand, the methods of asymptotic representation are adapted and have been generally applied. Though the literature covering investigations of this latter type is large it can hardly be said that the results recorded are by any means complete. Restrictions upon the range of the parameters are generally made and frequently only the forms of the Mathieu functions, i.e., of the solutions with the period $2 \pi$, are considered. Again, when forms asymptotic with respect to one parameter are obtained their dependence upon the remaining secondary parameter may not be considered, the results being established, therefore, only for a fixed configuration of the parameters relative to each other. Finally the investigations have almost exclusively been restricted to the case of a real variable. The most recent report on the status of the theory* says on this point: "While we believe that the theory of the Hill and Mathieu differential equations with real variables and parameters has to a certain extent been rounded out, it is to be emphasized that no such assertion can be made concerning these equations with complex variables and parameters.... Only when the problems bearing upon this point have been adequately treated may it be hoped to round out the theory of the Lamé equation as has been done in the case of the equation of Mathieu. Such an investigation would not only throw new light upon many differential equations of mathematical physics, but would make possible the application of certain of the functions obtained to problems of practical importance."

The present investigation is devoted to a general consideration of the asymptotic solutions of the Mathieu equation over the complex plane and for all real configurations of the parameter values in which at least one is

\footnotetext{
* Strutt, loc. cit. (Vorwort).
} 
numerically large. The analytic forms which represent the solutions asymptotically are found to differ in essentially different parameter configurations, while in its dependence upon the variable such a representation even for a specific solution and with one and the same configuration of parameters requires the employment of a variety of analytic forms. In general a special form is required for the description in the neighborhood of any point in which the coefficient of the equation vanishes, while outside such neighborhoods several forms again are made necessary by the incidence of the Stokes' phenomenon.

The limitation of the discussion to real parameter values was imposed to keep the extent of the investigation within its present bounds. The method in no way requires such a restriction.* In the matter of the method the present paper is based upon earlier papers of the author $\dagger$ which gave a general derivation of the asymptotic solutions of differential equations of the type

$$
\frac{d^{2} u}{d z^{2}}+\left\{\rho^{2} \chi_{0}^{2}(z)+\rho \chi_{1}(z)+\chi_{2}(z, \rho)\right\} u=0,
$$

in which $\rho$ is a large complex parameter and the coefficient $\chi_{0}^{2}(z)$ vanishes at some point of the domain considered. Aside from the considerations peculiar to the Mathieu equation, however, the presence of two independent parameters makes of the present discussion something more than a specialization of the general theory cited. With one parameter assigned to a primary role it must be shown that the hypotheses of the theory cited are met uniformly with respect to the secondary parameter which has remained free. This is essential to assure the uniform validity of the conclusions, i.e., that the degree of approximation afforded by the asymptotic representation is maintained during a variation of the parameters within the bounds of a given configuration.

By way of arrangement there have been grouped in chapter 1 such general considerations as are to be subsequently available. Of the following chapters each is given to the deductions peculiar to a specific configuration of parameters. Throughout the paper the forms of two fundamental pairs of solutions are deduced. This is desirable because of the fact that the members of any one pair of solutions may and do become asymptotically indistinguishable in certain regions of the complex plane. Aside from the general asymp-

* An analogous application of the method to a study of the Bessel functions with both the variable and the parameter complex was made by the author in the papers cited below.

$\dagger$ These Transactions, as follows: On the asymptotic solutions of ordinary differential equations, etc., vol. 33 (1931), p. 23; On the asymptotic solutions of differential equations, etc., vol. 34 (1932), p. 447; The asymptotic solutions of certain linear ordinary differential equations of the second order, vol. 36, p. 90. These papers will be referred to in the text by the designations $L_{1}, L_{2}$ and $L_{3}$. 
totic forms the special forms which apply to real values of the variable are noted, and the forms of the solutions of the associated Mathieu equation,

$$
\frac{d^{2} v}{d z^{2}}+\{\Omega \cosh 2 z-\Delta\} v=0,
$$

are deduced. The asymptotic equations for the characteristic values are given, and the characteristic exponent is asymptotically determined.

\section{Chapter 1}

\section{GENERAL CONSIDERATIONS}

1.1. The parameter configurations. The effect of replacing the variable $z$ by $z+\pi / 2$ in the equation (1) is merely to alter the sign of the cosine function, i.e., to replace the parameter $\Omega$ by its negative. There is, therefore, no loss of generality in assuming, as will henceforth be done, that $\Omega$ ranges only over the positive values and zero. The parameter $\Delta$, on the other hand, is to range unrestrictedly over all real values.

For any positive $\Omega$, however small it may be, the term $\Omega \cos 2 z$ becomes dominant over $\Delta$ when $z$ reaches a domain sufficiently remote from the axis of reals. In any such domain therefore the character of the differential equation is essentially altered if $\Omega$ is replaced by zero, and it may accordingly be expected that formulas which are to be valid uniformly for $\Omega \geqq 0$ may be obtained only for regions of the $z$ plane in which $|\vartheta(z)|$ is bounded. This fact suggests the grouping into separate configurations of those sets of parameter values in which $\Omega$ is relatively small. They are indicated as II and IX in Figure 1 below, the precise specifications to be later determined.

When $\Omega>0$, the function $\{\Delta-\Omega \cos 2 z\}$ vanishes at an infinite set of points in the complex plane. As $z$ moves at a suitable distance about any such point the asymptotic forms which represent a given solution of the differential equation must be altered, i.e., replaced by others, at certain specifiable intervals. This so called Stokes' phenomenon depends quantitatively upon the order of the zero which is encircled, and since this order changes from the first to the second when $\Omega$ and $|\Delta|$ become equal, it may be expected that results obtained on the assumption that the parameters are sufficiently different in numerical value may not remain uniformly valid when these values are allowed to approach equality. This fact serves as the motivation for considering as distinct configurations those indicated in Figure 1 by the designations IV and VII, in which the parameters numerically approximate each other. They will be precisely defined at appropriate points in the discussion which follows. The division of the half-plane of the coordinates $(\Delta, \Omega)$ into configurations is, therefore, such as is indicated in the 
figure, the hypothesis that at least one parameter be large having the effect of excluding from consideration a neighborhood of the point $O$.

1.2. The hypotheses of the general theory. The differential equation (1) may be transformed in a variety of ways into an equation of the general form

$$
\frac{d^{2} u}{d s^{2}}+\left\{\rho^{2} \chi_{0}^{2}(s, \sigma)+\rho \chi_{1}(s, \sigma)\right\} u=0,
$$

in which $\rho$, the primary parameter, and $\sigma$, the secondary parameter, are expressible in terms of $\Delta$ and $\Omega$. The particular substitutions and hence the particular equations which result are to depend upon the parameter configuration which obtains, and will therefore be made at appropriate points as the discussion proceeds.

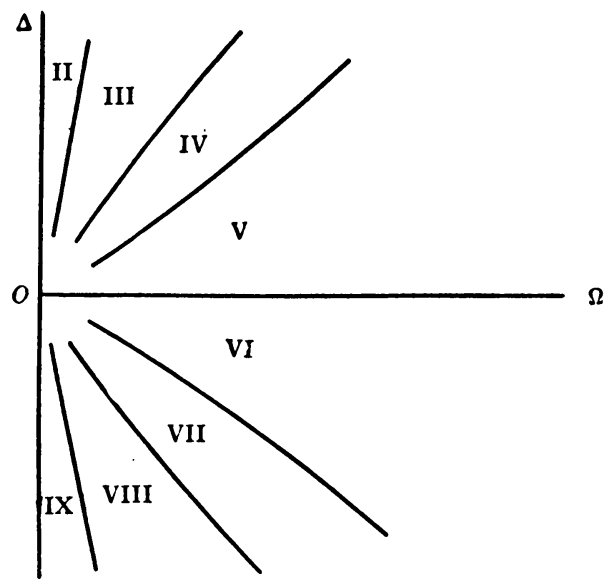

FIG. 1

Equations of the type (3) in which, however, the parameter $\sigma$ is absent (i.e., fixed) are familiar, the asymptotic forms of their solutions having been deduced* under hypotheses which for the present purposes may be enumerated in the following way:

(i) The range of the complex variable $s$ is to be a region $R_{s}$ in which the functions

$$
\left(s-s_{0}\right)^{-\nu} \chi_{0}^{2}(s) \text { and } \chi_{1}(s)
$$

are analytic, $s_{0}$ being some point of $R_{s}$ and $\nu$ being some real non-negative constant. Except in some fixed neighborhood of $s_{0}$ the several functions

(4) $\left\{\chi_{0}(s)\right\}^{-1},\left\{\int_{s_{0}}^{s} \chi_{0} d s\right\}^{-1},\left\{\chi_{1}(s) / \chi_{0}^{2}(s)\right\},\left\{\int\left(\chi_{1} / \chi_{0}\right) d s\right\}\left\{\int_{s_{0}}^{s} \chi_{0} d s\right\}^{-1}$ are to be bounded.

* Papers $L_{2}$ and $L_{3}$ cited above. In the formulas of paper $L_{3}$ the variables $\lambda, \chi_{1}, \phi$, and $\xi$ must be replaced by. $i_{\rho}, i_{\chi_{1}}, 2 \phi$ and $2 i \xi$ respectively in order that they may appear as given here. 
It is convenient to have at hand the following definitions:

(5)

$$
k=\frac{-i \chi_{1}\left(s_{0}\right)}{4 \chi_{0}{ }^{\prime}\left(s_{0}\right)}, \quad \eta(s)=\frac{i \chi_{1}(s)}{\chi_{0}(s)}+\frac{2 k \chi_{0}(s)}{\int_{s_{0}}^{s} \chi_{0} d s},
$$

$$
\phi(s)=\chi_{0}(s)-\frac{i \eta(s)}{2 \rho}, \quad \Phi(s)=\int_{\varepsilon_{0}}^{s} \phi(s) d s, \quad \xi=\rho \Phi .
$$

It follows then, as may be shown, from the hypothesis (i) that the functions

$$
\begin{aligned}
\omega(\phi) & \equiv \frac{3}{4}\left(\frac{\phi^{\prime}}{\phi}\right)^{2}-\frac{1}{2}\left(\frac{\phi^{\prime \prime}}{\phi}\right)-\frac{\nu(\nu+4)}{4(\nu+2)^{2}}\left(\frac{\phi}{\Phi}\right)^{2}, \\
\omega_{1} & \equiv \frac{\eta^{2}}{4}+\frac{k \chi_{0}^{2} \int_{80}^{s} \eta d s}{\Phi \int_{00}^{8} \chi_{0} d s}-\frac{k\left(4 \eta \chi_{0}-i \eta^{2} \rho^{-1}\right)}{2 \Phi}, \\
\Psi & \equiv \frac{\Phi^{\nu /(2 v+4)}}{\phi^{1 / 2}}
\end{aligned}
$$

are continuous in the region $R_{8}$ inclusive of the point $s=s_{0}$. A second and third hypothesis* made are the following:

(ii) The differential equation (3) is to be in normal form, i.e., such that either $\chi_{1} \equiv 0$ or else $\nu=2$ and

$$
\left\{3 x_{0}^{\prime} \chi_{1}^{\prime}-2 x_{0}^{\prime \prime} x_{1}\right\}_{s=s_{0}}=0 .
$$

(iii) Either the region $R_{8}$ is to be bounded, or else there are to exist constants $M$ and $H$ such that the relations

$$
\int\left|\frac{\omega(\phi)}{\phi} d s\right|<M, \quad \int\left|\frac{\omega_{1}(s)}{\phi} d s\right|<M
$$

are satisfied for all arcs of integration in $R_{s}$ on which $\left|s-s_{0}\right|>B$ and on which $\vartheta(\xi)$ varies monotonically with $|\xi|$.

When the secondary parameter $\sigma$ is not fixed but is permitted to vary, the formulas to be taken from the theory cited will be valid uniformly only if the hypotheses stated are satisfied uniformly with respect to $\sigma$. Specifically the functions (4) must be uniformly bounded in $R_{s}$, the functions (6) must be uniformly bounded in any fixed finite part of $R_{s}$, and the hypothesis (iii) must be fulfilled with constants $M$ and $H$ which are independent of $\sigma$.

* The hypothesis (iv) of papers $\mathrm{L}_{2}$ and $\mathrm{L}_{3}$ is not repeated here. It is obviously satisfied in every case of the present discussion. 
1.3. The solutions. When the equation (3) satisfies the several hypotheses and the primary parameter $\rho$ is sufficiently large, the relation defining the variable $\xi$ determines a map of the region $R_{\mathrm{s}}$ upon a corresponding region $R_{\xi}$ in the complex $\xi$ plane. This map is conformal except possibly at the point corresponding to $s_{0}$ where, if $\nu \neq 0$, the region $R_{\xi}$ has a branch point whose order depends upon $\nu$.

The relations

$$
\Xi^{(l)}:(l-1) \pi+\epsilon \leqq \arg \xi \leqq(l+1) \pi-\epsilon,
$$

with $l$ an integral index and $\epsilon$ an arbitrarily small but fixed positive constant, define in the domain $R_{\xi}$ the (overlapping) sub-regions $\Xi^{(l)}$. These correspond to respective sub-regions of $R_{\mathrm{s}}$ which will likewise be denoted by $\Xi^{(l)}$.

For any index $h$ the differential equation (3) possesses a fundamental pair of solutions $u_{h, 1}(s), u_{h, 2}(s)$, which are characterized by the fact that they are of peculiarly simple asymptotic forms as compared with the general solution for values of $s$ which are in the corresponding sub-region $\Xi^{(h)}$ and which are not too near the point $s_{0}$. When $s$ passes the bounds of the subregion $\Xi^{(h)}$ this simplicity is lost and devolves upon a new set of solutions which are in turn associated in the manner indicated with the new sub-region in which $s$ is then to be found. If $\nu \neq 0$ the forms referred to give valid representations of the respective solutions only so long as $|\xi| \geqq N$, where $N$ is a constant whose magnitude is determined by the degree of approximation which the asymptotic representation is required to aford. The excepted region $|\xi| \leqq N$ corresponds in $R_{8}$ to a neighborhood of the point $s_{0}$, and in this region a distinct representation must in general be employed.

The solutions $u_{h, j}(s), j=1,2$, with a particular index $h$ are thus because of their simplicity especially adapted for use in any deduction in which the associated region $\Xi^{(h)}$ plays a peculiar role. In terms of them, however, any other solutions may be simply expressed. In particular, it will be noted that if the point $z_{a}$ corresponds to $s_{a}$ under the correspondence of the variables which relates the equations (1) and (3), then the principal solutions $u(z)$, $U(z)$, of the equation (1) relative to $z_{a}$, i.e., those determined by the values

$$
u\left(z_{a}\right)=0, \frac{d u\left(z_{a}\right)}{d z}=1, \quad U\left(z_{a}\right)=1, \frac{d U\left(z_{a}\right)}{d z}=0,
$$

are given by the formulas

$$
\begin{aligned}
& u=\left(\frac{d z}{d s}\right)_{s=s_{a}}\left\{\frac{u_{h, 2}\left(s_{a}\right) u_{h, 1}(s)-u_{h, 1}\left(s_{a}\right) u_{h, 2}(s)}{W}\right\}, \\
& U=-\left\{\frac{u_{h, 2}^{\prime}\left(s_{a}\right) u_{h, 1}(s)-u_{h, 1}^{\prime}\left(s_{a}\right) u_{h, 2}(s)}{W}\right\},
\end{aligned}
$$


in which $h$ may be any index, the primes denote differentiation with respect to $s$, and $W$ designates the Wronskian

$$
W=u_{h, 1}^{\prime}(s) u_{h, 2}(s)-u_{h, 2}^{\prime}(s) u_{h, 1}(s),
$$

which is a constant.

The principal solutions relative to the origin $\left(z_{a}=0\right)$ will be designated throughout the discussion by $u_{o}(z)$ and $u_{e}(z)$. Inasmuch as the coefficient of the differential equation is an even function, they will be respectively odd and even functions of $z$ as is to be indicated by the subscripts chosen. The principal solutions relative to the point $z_{a}=\pi / 2$ will be denoted by $u_{\alpha}(z)$ and $u_{\beta}(z)$.

1.4. The asymptotic solutions when $\nu=1$. The special case of most frequent occurrence in the discussion which follows is that in which $\nu=1$, i.e., in which the zero of the coefficient $\chi_{0}{ }^{2}(s)$ is a simple one. It is convenient, therefore, to note at this point for general reference the specific formulas which then apply in the relations of the preceding section, in so far as they are later to be used. Thus, for $h=-1,0,1,2$ the solutions $u_{h, j}(s)$ are described by the following formulas:

When $|\xi| \geqq N$ and $s$ is in $\Xi^{(l)}$,

$$
u_{h, j}(s)=\rho^{-1 / 6} \phi^{-1 / 2}\left\{A_{j, 1}^{h, l} e^{i \xi}+A_{j, 2}^{h, l} e^{-i \xi}\right\}, j=1,2,
$$

\begin{tabular}{|c|c|c|c|c|c|c|c|c|c|c|c|c|c|}
\hline$(h, l)$ & $(-1,-1)$ & $(-1,0)$ & $(-1,1)$ & $(0,-1)$ & $(0,0)$ & $(0,1)$ & $(1,-1)$ & $(1,0)$ & $(1,1)$ & $(2,-1)$ & $(2,0)$ & $(2,1)$ & $(2,2)$ \\
\hline$\overline{A_{1,1}^{\mathbf{A}, l}}$ & [1] & {$[1]$} & 0 & [1] & $\overline{[1]}$ & [1] & [1] & [1] & {$[1]$} & 0 & 0 & [1] & [1] \\
\hline$\overline{A_{1,2}^{A} ! l}$ & 0 & {$[i]$} & {$[i]$} & $\overline{[-i]}$ & 0 & 0 & {$[-i]$} & 0 & 0 & $\overline{[-i]}$ & {$[-i]$} & {$[-i]$} & 0 \\
\hline 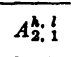 & 0 & 0 & {$[i]$} & 0 & 0 & $\overline{[i]}$ & {$[-i]$} & {$[-i]$} & 0 & {$[-i]$} & {$[-i]$} & 0 & 0 \\
\hline$A_{2}^{A_{2}^{2}, \frac{1}{2}}$ & [1] & [1] & [1] & [1] & [1] & {$[1]$} & 0 & [1] & {$[1]$} & 0 & [1] & [1] & [1] \\
\hline
\end{tabular}

with coefficients to be obtained from the following table:

and, when $|\xi| \leqq N$,

$$
u_{h, j}(s)=(2 \pi / 3)^{1 / 2} \Psi e^{(3 / 2-j) \pi i / 2}\left[\gamma_{1, j}^{(h)} \xi^{1 / 3} J_{-1 / 3}(\xi)+\gamma_{2, j}^{(h)} \xi^{1 / 3} J_{1 / 3}(\xi)\right],
$$

with the coefficients

\begin{tabular}{c|r|c|c|c|}
$h$ & -1 & 0 & 1 & 2 \\
\hline$\gamma_{1,1}^{(a)}$ & 1 & $e^{-\pi i / 3}$ & $e^{-\pi i / 3}$ & $e^{-2 \pi i / 3}$ \\
\hline$\gamma_{2,1}^{(),}$ & 1 & $e^{\pi i / 3}$ & $e^{\pi i / 3}$ & $e^{2 \pi i / 3}$ \\
\hline$\gamma_{1,2}^{(\lambda)}$ & $e^{\pi i / 3}$ & $e^{\pi i / 3}$ & 1 & 1 \\
\hline$\gamma_{2,2}^{(k)}$ & $e^{-\pi i / 3}$ & $e^{-\pi i / 3}$ & 1 & 1 \\
\hline
\end{tabular}


The symbols $J$ in these formulas designate Bessel functions in the familiar manner, and the symbol [ ] will be used throughout the discussion in the sense that $[Q]$ designates a quantity which differs from $Q$ by terms of the order of $\rho^{-1}$ and of the order of $N^{-1}$ uniformly in $\sigma$.

From formulas thus given the evaluations

$$
u_{h, 1}\left(s_{a}\right)=\frac{[1] e^{i \xi_{a}}}{\rho^{1 / 6} \phi_{a}^{1 / 2}}, \quad u_{h, 2}\left(s_{a}\right)=\frac{[1] e^{-i \xi_{a}}}{\rho^{1 / 6} \phi_{a}^{1 / 2}},
$$

when $\left|\xi_{a}\right| \geqq N$ and $\xi_{a}$ is in $\Xi^{(h)}$, and $W=[2 i] \rho^{2 / 3}$, will be immediately noted. Direct substitution in the relations (8b) leads, therefore, to the following formulas:

When $|\xi| \geqq N, z$ is in $\Xi^{(l)}$ and $z_{a}$ is in $\Xi^{(h)}$,

$$
\begin{aligned}
u= & \frac{[1]}{2 i}\left(\frac{d z}{d s}\right)_{s=s_{a}}\left(\frac{1}{\rho \phi_{a} \phi}\right)^{1 / 2}\left\{e^{-i \xi_{a}\left(A_{1,1}^{h, l} e^{i \xi}+A_{1,2}^{h, l} e^{-i \xi}\right)}\right. \\
& \left.\quad-e^{i \xi_{a}}\left(A_{2,1}^{h, l} e^{i \xi}+A_{2,2}^{h, l} e^{-i \xi}\right)\right\}, \\
U= & \frac{[1]}{2}\left(\frac{\phi_{a}}{\phi}\right)^{1 / 2}\left\{e^{-i \xi_{a}}\left(A_{1,1}^{h, l} e^{i \xi}+A_{1,1}^{h, l} e^{-i \xi}\right)+e^{i \xi a}\left(A_{2,1}^{h, l} e^{i \xi}+A_{2,2}^{h, l} e^{-i \xi}\right)\right\},
\end{aligned}
$$

and when $|\xi| \leqq N$ and $z_{a}$ is in $\Xi^{(h)}$,

$$
\begin{aligned}
u= & \left(\frac{d z}{d s}\right)_{s=s_{a}}\left(\frac{\pi}{6 \rho \phi_{a} \phi}\right)^{1 / 2} \xi^{1 / 6}\left\{e^{-i \xi_{a}-i \pi / 4}\left[\gamma_{1,1}^{(h)} \xi^{1 / 3} J_{-1 / 3}(\xi)+\gamma_{2,1}^{(h)} \xi^{1 / 3} J_{1 / 3}(\xi)\right]\right. \\
& \left.+e^{i \xi_{a}+\pi i / 4}\left[\gamma_{1,2}^{(h)} \xi^{1 / 3} J_{-1 / 3}(\xi)+\gamma_{2,2}^{(h)} \xi^{1 / 3} J_{1 / 3}(\xi)\right]\right\}, \\
U= & \left(\frac{\pi \phi_{a}}{6 \phi}\right)^{1 / 2} \xi^{1 / 6}\left\{e^{i \xi_{a}+\pi i / 4}\left[\gamma_{1,1}^{(h)} \xi^{1 / 3} J_{-1 / 3}(\xi)+\gamma_{2,1}^{(h)} \xi^{1 / 3} J_{1 / 3}(\xi)\right]\right. \\
& \left.+e^{i \xi_{a}-\pi i / 4}\left[\gamma_{1,2}^{(h)} \xi^{1 / 3} J_{-1 / 3}(\xi)+\gamma_{2,2}^{(h)} \xi^{1 / 3} J_{1 / 3}(\xi)\right]\right\} .
\end{aligned}
$$

From these forms certain terms, depending upon the indices, may under certain conditions be omitted as asymptotically negligible in comparison with others. The precise evaluations will be deferred to the points where applications of the formulas are to be made.

1.5. The "associated" Mathieu equation. The associated Mathieu equation (2) is obtainable from the equation (1) by substituting in the latter $i z$ in place of the variable $z$. Its solutions may, therefore, be derived from those discussed above by this simple change of variable. In particular it may be observed that the principal solutions relative to the origin, to be denoted by $v_{o}(z)$ and $v_{e}(z)$, are respectively odd and even functions of $z$, and that they are given by the formulas 


$$
\begin{aligned}
& v_{o}(z) \equiv-i u_{o}(i z), \\
& v_{e}(z) \equiv u_{e}(i z) .
\end{aligned}
$$

1.6. The solutions for generai values of $z$. The hypotheses stated in $\$ 1.2$ under which the forms of the solutions of the equation (1) are obtainable through the medium of the equation (3) restrict the variable to a region $R_{z}$ in which the coefficient $(\Delta-\Omega \cos 2 z)$ has at most one zero. It will be found in the subsequent discussion that this region over which the forms are directly deducible is in each case either the strip

$$
0 \leqq x \leqq \pi / 2 \text {, where } z=x+i y,
$$

or some closely related domain. It remains, therefore, to consider the extension of the asymptotic representations over the remaining parts of the $z$ plane. A method by which this may be done is to be outlined as follows.

Since the coefficient of the differential equation is an even periodic function with the period $\pi$, the function $u(n \pi-z)$ is a solution whenever $u(z)$ is such and $n$ is an integer. Hence each member of the several relations

$$
\begin{aligned}
& u_{o}(z) \equiv-u_{o}(\pi-z)+2 u_{o}(\pi / 2) u_{\beta}(\pi-z), \\
& u_{e}(z) \equiv-u_{e}(\pi-z)+2 u_{e}(\pi / 2) u_{\beta}(\pi-z), \\
& u_{o}(z) \equiv u_{o}(\pi-z)-2 u_{o}^{\prime}(\pi / 2) u_{\alpha}(\pi-z), \\
& u_{e}(z) \equiv u_{e}(\pi-z)-2 u_{e}^{\prime}(\pi / 2) u_{\alpha}(\pi-z)
\end{aligned}
$$

is a solution of the differential equation. The identities are established, therefore, by the fact that in each relation both members and likewise their derivatives take the same values at the point $z=\pi / 2$. A similar comparison of values at the point $z=2^{p} \pi$, whatever the integer $p$, establishes the further relations

$$
\begin{aligned}
& u_{o}(z) \equiv-u_{o}\left(2^{p+1} \pi-z\right)+2 u_{o}\left(2^{p} \pi\right) u_{e}\left(z-2^{p} \pi\right), \\
& u_{\theta}(z) \equiv u_{\theta}\left(2^{p+1} \pi-z\right)+2 u_{o}^{\prime}\left(2^{p} \pi\right) u_{o}\left(z-2^{p} \pi\right) .
\end{aligned}
$$

Let it be supposed now that the forms of the solutions have been deduced and so are known for all values of the variable which lie in the strip (13). It is to be shown then by the method of induction that they are deducible over the strip $S_{p}$ where $p$ is any integer and $S_{p}$ is defined by the relation

$$
S_{p}: \quad 0 \leqq x \leqq 2^{p} \pi .
$$

To begin with, let $z$ lie in the region $S_{0}$. Then either $z$ or $\pi-z$ lies in the strip (13). In the former case the representations of $u_{o}(z)$ and $u_{e}(z)$ are known by hypothesis, whereas in the latter they are given by the identities (14) in which the forms of the right-hand members are known. Proceeding, let the 
representations be considered known in the region $S_{p}$ with any specific $p$, and let $z$ lie in the strip $S_{p+1}$. Then either $z$ lies in $S_{p}$ and the forms are already known, or else both the values $\left(2^{p+1} \pi-z\right)$ and $\left(z-2^{p} \pi\right)$ lie in $S_{p}$ and the forms of the right-hand members of the relations (15) are known. In the latter event the identities furnish the representations sought in the part of $\delta_{p+1}$ not included in $\boldsymbol{S}_{p}$.

Finally the odd and even functional characters of the solutions $u_{o}(z)$, $u_{e}(z)$ may be drawn upon to extend their representations into the left-hand half-plane, and with the forms of these solutions at hand the representations of $u_{\alpha}(z)$ and $u_{\beta}(z)$ may be drawn from the identities (14).

1.7. The characteristic values. With any given value of $\Omega$ there are known to be associated specific characteristic values of $\Delta$ for which the differential equation (1) admits a periodic solution with the period $2 \pi$. These periodic solutions are enumerable, and are each either an odd or an even function of $z$. With a scheme of enumeration which will become clear as the subsequent quantitative discussion proceeds, the characteristic values for which the odd solution $u_{o}(z)$ has the period $2 \pi$ will be denoted by $S_{n}(\Omega)$, while those for which the period occurs in the even solution $u_{e}(z)$ will be designated by $C_{n}(\Omega)$. The equations of which these values are the roots are called characteristic equations.

Consider the characteristic equations for the values $S_{n}(\Omega)$. From the identity (15a) it is seen at once that a necessary and sufficient condition that $2 \pi$ be a period of $u_{o}(z)$ is that $u_{o}(\pi)=0$, an equation which in virtue of the relation (14c), with $z=\pi$, may be written

$$
u_{o}^{\prime}(\pi / 2) u_{\alpha}(0)=0 .
$$

If the root in question is one for which the factor $u_{o}^{\prime}(\pi / 2)$ vanishes, it follows from the identity (14c) that $u_{o}(z)$ admits no smaller period than $2 \pi$. On the other hand, if the root is one for which $u_{\alpha}(0)$ is zero, then the solutions $u_{o}(z)$ and $u_{\alpha}(z)$ are linearly dependent. It follows that $u_{o}(z)$ vanishes at $z=\pi / 2$, and hence from the relation (14a) that $u_{o}(z)$ admits the period $\pi$. With the enumeration to be chosen the characteristic equations for odd periodic solutions are accordingly the following:

$$
\begin{aligned}
u_{o}(\pi / 2)= & 0, \text { roots } S_{2 n}(\Omega), \\
& u_{o}(z) \text { periodic with the primitive period } \pi ; \\
u_{o}^{\prime}(\pi / 2)= & 0, \text { roots } S_{2 n+1}(\Omega), \\
& u_{o}(z) \text { periodic with the primitive period } 2 \pi .
\end{aligned}
$$

\footnotetext{
* Cf. Whittaker and Watson, loc. cit., \$19.2.
} 
The characteristic equations for even solutions may be similarly deduced. Thus from the identity ( $15 \mathrm{~b})$, with $p=0$, the condition that $2 \pi$ be a period of $u_{\theta}(z)$ is seen to be $u_{e}^{\prime}(\pi)=0$. From the derived relation (14b), taken at $z=\pi$, the condition is found to be

$$
u_{\epsilon}(\pi / 2) u_{\beta}^{\prime}(0)=0 .
$$

If for the root in question $u_{e}(\pi / 2)$ is zero, the identity (14b) shows that a smaller period than $2 \pi$ is precluded. In the alternative the factor $u_{\beta}{ }^{\prime}(0)$ is zero, $u_{e}(z)$ and $u_{\beta}(z)$ are dependent and hence $u_{e}^{\prime}(z)$ vanishes at $z=\pi / 2$. It follows from the relation $(14 \mathrm{~d})$ then that $u_{e}(z)$ admits the period $\pi$. In this instance, therefore, the characteristic equations are

$$
u_{e}^{\prime}(\pi / 2)=0, \text { roots } C_{2 n}(\Omega),
$$

$u_{e}(z)$ periodic with the primitive period $\pi$;

$$
u_{\odot}(\pi / 2)=0 \text {, roots } C_{2 n+1}(\Omega) \text {, }
$$

$u_{e}(z)$ periodic with the primitive period $2 \pi$.

1.8. The Mathieu functions. When $\Delta$ is a characteristic value $S_{n}(\Omega)$ or $C_{n}(\Omega)$, the corresponding periodic solution $u_{o}(z)$ or $u_{e}(z)$ is after suitable normalization known as a Mathieu function, and is respectively designated by $\operatorname{se}_{n}(z, \Omega)$ or $\operatorname{ce}_{n}(z, \Omega)$. Two modes of normalization have been commonly employed. The first* uses the stipulation that the coefficients of $\sin n z$ and $\cos n z$ in the respective Fourier expansions of $\operatorname{se}_{n}(z, \Omega)$ and $\operatorname{ce}_{n}(z, \Omega)$ be unity, i.e.,

$$
\begin{aligned}
& \frac{1}{\pi} \int_{-\pi}^{\pi} \mathrm{se}_{n}(x, \Omega) \sin n x d x=1, \\
& \frac{1}{\pi} \int_{-\pi}^{\pi} \mathrm{ce}_{n}(x, \Omega) \cos n x d x=1+\delta_{0, n}, \quad \delta_{0, n}=\left\{\begin{array}{l}
1 \text { for } n=0, \\
0 \text { for } n \neq 0 .
\end{array}\right.
\end{aligned}
$$

Since the integrands in these relations are even functions, the intervals of integration may, of course, be reduced to $(0, \pi)$. It may, however, be further observed that in virtue of the equations $(17) ;(18)$, and (14),

$$
\begin{aligned}
& \operatorname{se}_{n}(z, \Omega) \equiv(-1)^{n+1} \operatorname{se}_{n}(\pi-z, \Omega), \\
& \operatorname{ce}_{n}(z, \Omega) \equiv(-1)^{n} \operatorname{ce}_{n}(\pi-z, \Omega),
\end{aligned}
$$

i.e., the Mathieu functions are each either even or odd in the variable $z-\pi / 2$. The ranges of integration above may, therefore, be reduced further to $(0, \pi / 2)$, the formulas which result being

* Cf. Whittaker and Watson, loc. cit. 
(20)

$$
\begin{aligned}
& \operatorname{se}_{n}(z, \Omega)=\left\{\frac{\pi u_{0}(z)}{4 \int_{0}^{\pi / 2} u_{0}(x) \sin n x d x}\right\}_{\Delta-S_{n}(\Omega)} \\
& \operatorname{ce}_{n}(z, \Omega)=\left\{\frac{\pi u_{e}(z)}{\left(4-2 \delta_{0, n}\right) \int_{0}^{\pi / 2} u_{e}(x) \cos n x d x}\right\}_{\Delta-C_{n}(\Omega)}
\end{aligned}
$$

A second mode of normalization* is based on the requirements

$$
\frac{1}{\pi} \int_{-\pi}^{\pi} \operatorname{se}_{n}^{2}(x, \Omega) d x=1, \frac{1}{\pi} \int_{-\pi}^{\pi} \operatorname{ce}_{n}^{2}(x, \Omega) d x=1+\delta_{0, n} .
$$

In this case the formulas obtained are

$$
\begin{aligned}
\operatorname{se}_{n}(z, \Omega) & =\left\{\frac{\pi^{1 / 2} u_{0}(z)}{2\left(\int_{0}^{\pi / 2} u_{0}^{2}(x) d x\right)^{1 / 2}}\right\}_{\Delta-S_{n}(\Omega)} \\
\operatorname{ce}_{n}(z, \Omega) & =\left\{\frac{\pi^{1 / 2} u_{e}(z)}{2^{1-\delta_{0, n} / 2}\left(\int_{0}^{\pi / 2} u_{e}^{2}(x) d x\right)^{1 / 2}}\right\}_{\Delta-C_{n}(\Omega)}
\end{aligned}
$$

1.9. Other periodic solutions. The characteristic equations for values of $\Delta$ which yield periodic solutions with periods other than $\pi$ or $2 \pi$ may be deduced by considerations similar to those of $\$ 1.7$. The identities
(a) $u(z) \equiv-u(2 p \pi-z)+2 u(p \pi) u_{\theta}(p \pi-z)$,
(b) $u(z) \equiv u(2 p \pi-z)-2 u^{\prime}(p \pi) u_{0}(p \pi-z)$,
(c) $u(z) \equiv-u((2 p-1) \pi-z)+2 u\left(\left(p-\frac{1}{2}\right) \pi\right) u_{\beta}(p \pi-z)$,
(d) $u(z) \equiv u((2 p-1) \pi-z)-2 u^{\prime}\left(\left(p-\frac{1}{2}\right) \pi\right) u_{\alpha}(p \pi-z)$.

are easily verified when $p$ is any integer and $u(z)$ is an arbitrary solution of the differential equation. With the use of them it can be shown, as is outlined below, that periodic solutions with the periods indicated occur for values of $\Delta$ which are roots of the respective equations

$$
\begin{array}{cl}
u_{o}(n \pi / 2)=0, & \text { odd solutions with period } n \pi, \\
u_{o}^{\prime}(n \pi / 2)=0, & \text { odd solutions with period } 2 n \pi, \\
u_{o}^{\prime}(n \pi / 2)=0, & \text { even solutions with period } n \pi, \\
u_{e}(n \pi / 2)=0, & \text { even solutions with neriod } 2 n \pi .
\end{array}
$$

* Cf. Strutt, loc. cit. 
Moreover, if $n$ is the smallest integer for which an equation is satisfied, the period indicated is primitive.

Consider the equations (23). Their sufficiency for the indicated periodicities may be verified by observing that they imply through the pertinent identities (22) respectively that $u_{o}(z+n \pi)= \pm u_{o}(z)$. Conversely, if $2 n \pi$ is a period of $u_{o}(z)$, then $u_{o}(n \pi)=0$ by the identity (22a), and this leads when $n$ is even through the relation (22b) to the one or the other of the equations (23). If $n$ is odd the result follows from the identities (22c) and (22d), together with the fact that at least one of the solutions $u_{\alpha}(z)$ and $u_{\beta}(z)$ must differ from zero at the point $z=(n+1) \pi / 2$.

The necessity and sufficiency of the equations (24) for solutions of their associated types is proved similarly, though in some instances the identities (22) must be differentiated prior to their application.

1.10. The characteristic exponent. When the parameters $\Delta$ and $\Omega$ are both fixed the differential equation in general admits no periodic solution. In this case it is known from Floquet's theory of differential equations with simply periodic coefficients that there are two solutions of the forms

$$
e^{\mu z} \phi(z) \text {, and } e^{-\mu z} \phi(-z),
$$

in which $\phi(z)$ is a periodic function with the period $\pi$, while $\mu$, the so called characteristic exponent, is a constant which depends upon $\Delta$ and $\Omega$. The equation for $\mu$ is*

$$
e^{2 \pi \mu}-2 \Theta e^{\pi \mu}+1=0
$$

whence

$$
\mu=\frac{1}{\pi} \cosh ^{-1} \Theta=\frac{i}{\pi} \cos ^{-1} \Theta,
$$

with $\Theta=u_{e}(\pi)$. The alternative evaluation

$$
\Theta=2 u_{e}(\pi / 2) u_{\beta}(0)-1
$$

may be obtained from the relation (14b).

It is evident that $\mu$ is either real or pure imaginary according as $\Theta>1$ or $\Theta<1$. In the former case the solutions noted above become infinite near the one or the other extremity of the axis of reals and are called unstable; in the latter case they remain bounded for real values of $z$ and are called stable.

1.11. Certain elliptic integrals. It will be found now and again in the discussion which follows, that the comparison and identification of certain

* Cf. Horn, J., Gewöhnliche Differentialgleichungen, Leipzig, 1905, p. 242. 
superficially dissimilar formulas will depend upon the approximate or asymptotic evaluation of certain elliptic integrals of the type

$$
G\left(\tau, h^{2}\right)=\int_{0}^{\pi / 2} \frac{1-\tau \sin ^{2} \zeta}{\left\{1-h^{2} \sin ^{2} \zeta\right\}^{1 / 2}} d \zeta .
$$

The value of $h$ will in every case be either near zero or near 1 , and $\tau$ will be either 1 or $h^{2}$.

In terms of the standard complete elliptic integrals

$$
K=\int_{0}^{\pi / 2} \frac{d \zeta}{\left\{1-h^{2} \sin ^{2} \zeta\right\}^{1 / 2}}, \quad E=\int_{0}^{\pi / 2}\left\{1-h^{2} \sin ^{2} \zeta\right\} d \zeta
$$

it is evident that

$$
G\left(\tau, h^{2}\right)=K+\frac{\tau}{h^{2}}(E-K) .
$$

Hence on substituting for these integrals their expansions in powers of $h$, it is found that when $h^{2}$ is nearly zero

$$
\begin{aligned}
G\left(1, h^{2}\right) & =\frac{\pi}{4}\left\{1+\frac{h^{2}}{8}+h^{4} O(1)\right\}, \\
G\left(h^{2}, h^{2}\right) & =\frac{\pi}{2}\left\{1-\frac{h^{2}}{4}+h^{4} O(1)\right\} .
\end{aligned}
$$

On the other hand, when $h^{2}$ is nearly 1 the Landen Transformation*

$$
h \sin \zeta=\sin (2 t-\zeta)
$$

yields the form

$$
G\left(\tau, h^{2}\right)=\frac{-\tau}{h}+\frac{2}{1+h} \int_{0}^{t_{1}} \frac{\left(1-\frac{\tau}{h}\right)+\frac{2 \tau}{h} \cos ^{2} t}{\cos t\left\{1+\epsilon^{2} \tan ^{2} t\right\}^{1 / 2}} d t,
$$

in which

$$
t_{1}=\sin ^{-1}\left\{\frac{1+h}{2}\right\}^{1 / 2}, \quad \epsilon=\frac{1-h}{1+h} .
$$

The quantity $\epsilon^{2} \tan ^{2} t$ is uniformly small of the order of $\epsilon$. Hence the radical may be replaced by its binomial expansion, whereupon the integration leads to the formula

\footnotetext{
* Cf. Hancock, H., Elliptic Integrals, New York, 1917, p. 84.
} 


$$
\begin{aligned}
G\left(\tau, h^{2}\right)=\frac{-\tau}{h} & +\left(\frac{2}{1+h}\right)\left(\frac{2 \tau \sin t_{1}}{h}-\frac{\epsilon^{2}(h-\tau) \sin t_{1}}{4 h \cos ^{2} t_{1}}\right. \\
& \left.+\left\{\left(\frac{h-\tau}{2 h}\right)+\frac{\epsilon^{2}}{8}\left(1-\frac{5 \tau}{h}\right)\right\} \log \frac{1+\sin t_{1}}{1-\sin t_{1}}\right)+o\left(\epsilon^{2}\right) .
\end{aligned}
$$

For the special values of $\tau$ this reduces to.

$$
\begin{aligned}
& G\left(1, h^{2}\right)=\frac{-1}{h}+\frac{2}{h}\left(\frac{2}{1+h}\right)^{1 / 2}+\frac{1-h}{h(1+h)} \log \frac{1-h}{8}+O\left(\epsilon^{2} \log \epsilon\right), \\
& G\left(h^{2}, h^{2}\right)=-h+2 h\left(\frac{2}{1+h}\right)^{1 / 2}-\frac{1-h}{1+h} \log \frac{1-h}{8}+O\left(\epsilon^{2} \log \epsilon\right) .
\end{aligned}
$$

\section{Chapter 2}

\section{The CONFIGURATION II}

2.1. The differential equation. When the relative values of the parameters $\Delta$ and $\Omega$ are such that the point $(\Omega, \Delta)$ in Figure 1 lies in the region II at a sufficient distance from $O$, i.e., more specifically when $\Delta$ is large and positive, and with a constant $M_{1}$ (to be specified below) the relation

$$
0 \leqq \Omega \leqq \frac{1}{M_{1}} \Delta
$$

is fulfilled, the substitutions

$$
\rho=\Delta^{1 / 2}, \quad \sigma^{2}=\Omega / \Delta, \quad s=z^{*}
$$

give to the equation (1) the form (3) with

$$
\begin{aligned}
& \chi_{0} \equiv \phi, \quad \chi_{1} \equiv 0, \\
& \phi^{2} \equiv 1-\sigma^{2} \cos 2 s .
\end{aligned}
$$

Let the variable $z$ be restricted to any finite region of the complex plane. Then a number $M_{1}$ may be determined such that for all admitted values of $z$

$$
|y| \leqq \frac{1}{2} \cosh ^{-1} \frac{M_{1}}{2},
$$$$
z=x+i y \text {. }
$$

The constant $M_{1}$ of the relation (2.1), which determines the parameter values to be included in the present configuration, is to be one with which the condition (2.4a) is fulfilled. The primary parameter $\rho$ is to be thought of as

* The distinction between $s$ and $z$, which in the present instance is non-existent, is drawn for the purpose of making the formulas subsequently useful in a case when these variables are not the same. 
bounded below but not above, and the secondary parameter $\sigma$ is evidently restricted to the range

$$
0 \leqq \sigma^{2} \leqq \frac{1}{M_{1}}
$$

The relation $(2.4 \mathrm{a})$, together with

$$
0 \leqq x \leqq \frac{\pi}{2},
$$

defines a strip of the $z$ plane which is to be designated as $R_{z}$. The corresponding domain of the variable $s$ is

$$
R_{8}: \quad 0 \leqq s^{\prime} \leqq \pi / 2, \quad\left|s^{\prime \prime}\right| \leqq \frac{1}{2} \cosh ^{-1}\left(M_{1} / 2\right), \quad s=s^{\prime}+i s^{\prime \prime} .
$$

This region includes the origin and it is readily verified that with $s_{0}=0$ the hypothesis (i) of $\$ 1.2$ is fulfilled uniformly in $\sigma$ with $\nu=0$. The hypotheses (ii) and (iii) are likewise fulfilled, since $\chi_{1} \equiv 0$ and $R_{8}$ is bounded. From the formulas (5) it is seen that in the present instance $\eta(s) \equiv \omega_{1}(s) \equiv k=0$, in consequence of which

$$
\omega(\phi) \equiv 1+\frac{1}{4 \phi^{2}}-\frac{5\left(1-\sigma^{4}\right)}{4 \phi^{4}}, \quad \Psi \equiv \phi^{-1 / 2} .
$$

These functions are bounded uniformly in $\sigma$ and hence the requirements enumerated in $\$ 1.2$ are completely fulfilled.

2.2. The solutions. Since the case in hand is one in which $\nu=0$, there exist solutions of the differential equation which maintain a single asymptotic form over the entire region $R_{z}$. Such solutions with their respective forms are

$$
\begin{aligned}
& u_{0,1}(s)=\phi^{-1 / 2} e^{i \xi}[1], \\
& u_{0,2}(s)=\phi^{-1 / 2} e^{-i \xi}[1] .
\end{aligned}
$$

Their Wronskian has the value $W=[2 i] \rho$. The principal solutions relative to the point $z=0$ are accordingly computed directly from the formula (8b), with $h=0, s_{a}=0$, to be

with

$$
\begin{aligned}
& u_{o}(z)=\frac{1}{2 i}\left\{\frac{1}{\rho^{2} \phi_{1} \phi}\right\}^{1 / 2}\left\{e^{i \xi}[1]-e^{-i \xi}[1]\right\} \\
& u_{\theta}(z)=\frac{1}{2}\left\{\frac{\phi_{1}}{\phi}\right\}^{1 / 2}\left\{e^{i \xi}[1]+e^{-i \xi}[1]\right\}
\end{aligned}
$$

$$
\begin{aligned}
\rho \phi & =\{\Delta-\Omega \cos 2 z\}^{1 / 2}, \quad \rho \phi_{1}=\{\Delta-\Omega\}^{1 / 2}, \\
\xi & =\int_{0}^{z}\{\Delta-\Omega \cos 2 z\}^{1 / 2} d z .
\end{aligned}
$$


Inasmuch as

$$
e^{i \xi}[1]-e^{-i \xi}[1]=[2 i] \sin [\xi],
$$

with analogous formulas involving the other trigonometric functions, it is seen in particular that for real values of the variable

$$
u_{o}(x)=\frac{[1]}{\{(\Delta-\Omega)(\Delta-\Omega \cos 2 x)\}^{1 / 4}} \sin \left[\int_{0}^{x}\{\Delta-\Omega \cos 2 x\}^{1 / 2} d x\right],
$$

$$
u_{e}(x)=\left\{\frac{\Delta-\Omega}{\Delta-\Omega \cos 2 x}\right\}^{1 / 4}[1] \cos \left[\int_{0}^{x}\{\Delta-\Omega \cos 2 x\}^{1 / 2} d x\right] .
$$

The principal solutions relative to $z=\pi / 2$ are similarly found to be given by the formulas

with

$$
\begin{aligned}
& u_{\alpha}(z)=\frac{1}{2 i}\left\{\frac{1}{\rho^{2} \phi_{2} \phi}\right\}^{1 / 2}\left\{e^{i\left(\xi-\xi_{2}\right)}[1]-e^{-i\left(\xi-\xi_{2}\right)}[1]\right\} \\
& u_{\beta}(z)=\frac{1}{2}\left\{\frac{\phi_{2}}{\phi}\right\}^{1 / 2}\left\{e^{i\left(\xi-\xi_{2}\right)}[1]+e^{-i\left(\xi-\xi_{2}\right)}[1]\right\}
\end{aligned}
$$

$$
\rho \phi_{2}=\{\Delta+\Omega\}^{1 / 2}, \quad \xi-\xi_{2}=\int_{\pi / 2}^{z}\{\Delta-\Omega \cos 2 z\}^{1 / 2} d z
$$

When $z$ is real they are

$$
\begin{aligned}
& u_{\alpha}(x)=\frac{-[1]}{\{(\Delta+\Omega)(\Delta-\Omega \cos 2 x)\}^{1 / 4}} \sin \left[\int_{x}^{\pi / 2}\{\Delta-\Omega \cos 2 x\}^{1 / 2} d x\right] \\
& u_{\beta}(x)=\left\{\frac{\Delta+\Omega}{\Delta-\Omega \cos 2 x}\right\}^{1 / 4}[1] \cos \left[\int_{x}^{\pi / 2}\{\Delta-\Omega \cos 2 x\}^{1 / 2} d x\right] .
\end{aligned}
$$

In the special case that $\sigma=0$ (i.e., $\Omega=0$ ) the differential equation (1) is directly integrable, and it is verified immediately that the formulas above are correct when the symbols [ ] are omitted. It may be concluded, therefore, in the discussion of this chapter that the quantities [1] reduce to 1 when $\sigma^{2}=0$.

2.3. The solutions of the associated Mathieu equation. The principal solutions of the associated Mathieu equation (2) relative to the origin may be derived from the functions (2.8) by the substitutions (12) as was noted in \$1.5. Their forms so obtained are

$$
\begin{aligned}
& v_{o}(z)=\frac{[1]}{\{(\Delta-\Omega)(\Delta-\Omega \cosh 2 z)\}^{1 / 4}} \sinh \left[\int_{0}^{z}\{\Delta-\Omega \cosh 2 z\}^{1 / 2} d z\right], \\
& v_{e}(z)=\left\{\frac{\Delta-\Omega}{\Delta-\Omega \cosh 2 z}\right\}^{1 / 4}[1] \cosh \left[\int_{0}^{z}\{\Delta-\Omega \cosh 2 z\}^{1 / 2} d z\right],
\end{aligned}
$$


the region for $z$ being

$$
\begin{gathered}
|x| \leqq \frac{1}{2} \cosh ^{-1} \frac{M_{1}}{2}, \\
-\pi / 2 \leqq y \leqq 0 .
\end{gathered}
$$

The solutions (2.10) are evidently asymptotically multiples of each other when $z$ is real and large. A pair, $v_{\gamma}(z), v_{\delta}(z)$, not subject to this disadvantage is that obtainable by the substitution of $i z$ for $s$ from the functions (2.7). Their forms are explicitly

$$
\begin{aligned}
& v_{\gamma}(z)=\frac{[1]}{\{\Delta-\Omega \cosh 2 z\}^{1 / 4}} \exp \left[-\int_{0}^{z}\{\Delta-\Omega \cosh 2 z\}^{1 / 2} d z\right], \\
& v_{\delta}(z)=\frac{[1]}{\{\Delta-\Omega \cosh 2 z\}^{1 / 4}} \exp \left[\int_{0}^{z}\{\Delta-\Omega \cosh 2 z\}^{1 / 2} d z\right] .
\end{aligned}
$$

2.4. The characteristic values. If $S_{p}(\Omega)$ and $C_{q}(\Omega)$ are a pair of characteristic values, the substitution of the forms $(2.8 \mathrm{~b})$ into the characteristic equations (17) and (18) shows that each of these values is a root of an equation

$$
\left[\int_{0}^{\pi / 2}\{\Delta-\Omega \cos 2 x\}^{1 / 2} d x\right]=\frac{n \pi}{2}
$$

with the integer $n$ suitably adjusted to $p$ or $q$ as the case may be. To determine this adjustment, it need merely be observed that when $\Omega=0$ the equation reduces to $\Delta=n^{2}$, and the corresponding Mathieu functions to $\sin n z$ and $\cos n z$. Since these are by definition the forms of $\operatorname{se}_{n}(z, 0)$ and $\operatorname{ce}_{n}(z, 0)$, it must be concluded that $p=n$ and $q=n$, i.e., the form (2.12) is that of the characteristic equation both for $S_{n}(\Omega)$ and for $C_{n}(\Omega)$.

The symbol [ ] in the equation (2.12) represents a quantity of the order of $\Delta^{-1 / 2}$ uniformly in $\sigma$, which vanishes when $\sigma=0$. Since it like the equation (1) depends analytically upon $\sigma^{2}$, the equation (2.12) may be written

$$
\int_{0}^{\pi / 2}\{\Delta-\Omega \cos 2 x\}^{1 / 2} d x+\sigma^{2} O\left(\Delta^{-1 / 2}\right)=\frac{n \pi}{2} .
$$

The substitution $x=\pi / 2-\zeta$ reduces this to

$$
\Delta^{1 / 2}\left\{\left(1+\sigma^{2}\right)^{1 / 2} G\left(h^{2}, h^{2}\right)+\sigma^{2} O\left(\Delta^{-1 / 2}\right)\right\}=\frac{n \pi}{2},
$$


where $G$ is the elliptic integral of (26) with $h^{2}=2 \sigma^{2} /\left(1+\sigma^{2}\right)$. Since this value of $h^{2}$ is small, the evaluation (26a) gives to the equation the form

$$
\Delta^{1 / 2}\left\{1+\sigma^{4} O(1)+\sigma^{2} O\left(\Delta^{-1}\right)\right\}=n,
$$

from which it follows that

$$
\begin{aligned}
& S_{n}(\Omega)=n^{2}+\frac{\Omega}{n^{2}} O(1), \\
& C_{n}(\Omega)=n^{2}+\frac{\Omega}{n^{2}} O(1),
\end{aligned}
$$

the quantities indicated by the symbols $O(1)$ being uniformly bounded as to $n$ and $\Omega$ while the configuration with which the present chapter deals is maintained.

2.5. The characteristic exponent. The s ubstitution into the formula (25b) of the values given by $(2.8 \mathrm{~b})$ and $(2.9 \mathrm{~b})$ yields the evaluation

$$
\begin{aligned}
\Theta & =[2] \cos \left[\xi_{2}\right] \cos \left[\xi_{2}\right]-1 \\
& =\cos 2 \xi_{2}+\sigma^{2} O\left(\Delta^{-1 / 2}\right) .
\end{aligned}
$$

Accordingly, from (25a) an asymptotic formula for the characteristic exponent is

$$
u=\frac{i}{\pi} \cos ^{-1}\left\{\cos \left(\int_{0}^{\pi / 2} 2\{\Delta-\Omega \cos 2 x\}^{1 / 2} d x\right)+\frac{\Omega^{2}}{\Delta^{3 / 2}} O(1)\right\} .
$$

When $\Omega=0$ this reduces to $\mu=i \Delta^{1 / 2}$, a result which may be verified by actual integration of the differential equation.

Inasmuch as the quantity within the brace in the formula (2.14) does not exceed unity, except possibly for very small ranges of the parameters near those values for which the integral is a multiple of $\pi$, it follows that the configuration under consideration in this chapter is predominantly one of stable solutions.*

\section{Chapter 3}

\section{The CONFIgURATION III}

3.1. Definitions. The parameter configuration contiguous with that of the preceding chapter and designated by III in Figure 1 is to be defined by the relation

$$
\frac{1}{M_{1}} \Delta \leqq \Omega \leqq \Delta-M_{2} \Delta^{1 / 2}
$$

\footnotetext{
*Cf. the Figure 3 in Strutt, loc. cit.
} 
in which $M_{1}$ is the constant in (2.1), and $M_{2}$ is to be momentarily discussed. The substitutions

$$
\rho=\frac{\Delta-\Omega}{\Delta^{1 / 2}}, \quad \sigma^{2}=1-\frac{\Omega}{\Delta}, \quad s=\frac{-i z}{\sigma}
$$

reduce the differential equation (1) in this case to the form (3) with

$$
\begin{aligned}
& \chi_{0} \equiv \phi, \quad \chi_{1} \equiv 0, \\
& \phi^{2} \equiv 2\left(1-\sigma^{2}\right) \frac{\sinh ^{2} \sigma s}{\sigma^{2}}-1 .
\end{aligned}
$$

The parameter $\rho$ is evidently restricted by the relation $\rho \geqq M_{2}$, and since the degree of approximation which the asymptotic formulas yield depends upon the magnitude of $\rho$, the constant $M_{2}$ is in any specific case to be chosen such that representations which are uniformly suitable to the purposes intended are obtained. The secondary parameter is clearly confined to the fixed closed interval

$$
0 \leqq \sigma^{2} \leqq 1-\frac{1}{M_{1}},
$$

in which the lower boundary could in fact more strictly be replaced by $M_{2} \Delta^{-1 / 2}$.

Let $z$ be restricted for the discussion of this configuration to the infinite half-strip $R_{z}$ given by the formulas

$$
R_{z}: \quad-\pi / 2 \leqq x \leqq \pi / 2, \quad 0 \leqq y .
$$

The extension of the solutions from this domain to the entire strip (13) may be accomplished by the use of the identities

$$
\begin{aligned}
& u_{\alpha}(z) \equiv u_{\alpha}(-z)-2 u_{\alpha}^{\prime}(0) u_{o}(-z), \\
& u_{\beta}(z) \equiv-u_{\beta}(-z)+2 u_{\beta}(0) u_{e}(-z),
\end{aligned}
$$

and the odd and even characters of $u_{o}(z)$ and $u_{e}(z)$. Their extension to general values of $z$ thereupon follows on the lines of $\$ 1.6$.

3.2. The variables $s, \Phi$ and $\xi$. The region $R_{\varepsilon}$ corresponding to $R_{z}$ is the infinite half-strip

$$
R_{s}: \quad 0 \leqq s^{\prime}, \quad-\frac{\pi}{2 \sigma} \leqq s^{\prime \prime} \leqq \frac{\pi}{2 \sigma} .
$$

Within this region $\chi_{0}^{2}(s)$ has a single zero located on the axis of reals at the point

$$
s_{0}^{\prime} \equiv \frac{1}{\sigma} \sinh ^{-1} \frac{\sigma}{\left\{2\left(1-\sigma^{2}\right)\right\}^{1 / 2}} .
$$


Though $s_{0}^{\prime}$ depends upon $\sigma$ it is both bounded and bounded from zero for all admitted values of the parameters.

The relation between $s$ and the quantity $\Phi$ maps $R_{s}$ upon a corresponding region $R_{\Phi}$ conformally except at the point $s_{0}^{\prime}$. The shape of $R_{\Phi}$ may be easily determined by observing the values of $\Phi$ when $s$ is either real or on the boundaries of $R_{s}$. With $R_{s}$ thought of as cut along the axis of reals from the origin to $s_{0}^{\prime}$ these values for the upper half of $R_{\mathrm{s}}$ are

$$
\begin{aligned}
\text { for } s^{\prime \prime} & =0+\text { and } 0 \leqq s^{\prime} \leqq s_{0}^{\prime}, \\
\Phi & =e^{\pi i} \int_{s^{\prime}}^{s^{\prime}} i\left\{1-2\left(1-\sigma^{2}\right) \frac{\sinh ^{2} \sigma s^{\prime}}{\sigma^{2}}\right\}^{1 / 2} d s^{\prime} ; \\
\text { for } s^{\prime} & =0 \text { and } 0 \leqq s^{\prime \prime} \leqq \pi /(2 \sigma), \\
\Phi & =\Phi(0)+e^{\pi i / 2} \int_{0}^{s^{\prime \prime}} i\left\{2\left(1-\sigma^{2}\right) \frac{\sin ^{2} \sigma s^{\prime \prime}}{\sigma^{2}}+1\right\}^{1 / 2} d s^{\prime \prime} ; \\
\text { for } s^{\prime \prime} & =0 \text { and } s_{0}^{\prime} \leqq s^{\prime}, \\
\Phi & =\int_{o^{\prime}}^{\bullet^{\prime}}\left\{2\left(1-\sigma^{2}\right) \frac{\sinh ^{2} \sigma s^{\prime}}{\sigma^{2}}-1\right\}^{1 / 2} d s^{\prime} ; \\
\text { for } s^{\prime \prime} & =\pi /(2 \sigma) \text { and } 0 \leqq s^{\prime}, \\
\Phi & =\Phi\left(\frac{\pi i}{2 \sigma}\right)+\int_{0}^{\iota^{\prime}} i\left\{2\left(1-\sigma^{2}\right) \frac{\cosh ^{2} \sigma s^{\prime}}{\sigma^{2}}+1\right\}^{1 / 2} d s^{\prime} .
\end{aligned}
$$

The map of the lower half of $R_{\mathrm{s}}$ is obtainable by reflection from that of the upper half, since conjugate complex values of $s$ lead to conjugate values of $\Phi$.

Finally since

$$
\left|\frac{\sinh \sigma s}{\sigma}\right|>\frac{2}{\pi}|s|,
$$

it follows that when $|s|$ is sufficiently large

$$
\begin{aligned}
& \phi \sim\left\{2\left(1-\sigma^{2}\right)\right\}^{1 / 2} \frac{\sinh \sigma s}{\sigma}, \\
& \Phi \sim \frac{2}{\sigma^{2}}\left\{2\left(1-\sigma^{2}\right)\right\}^{1 / 2} \sinh ^{2} \frac{\sigma s}{2},
\end{aligned}
$$

the symbolism designating that the ratio of the members of either relation becomes 1 as $|s| \rightarrow \infty$. From the second relation it follows that when $c$ is any sufficiently large constant the line $s^{\prime}=c$ maps upon a simple curve in $R_{\Phi}$. The uniqueness of the correspondence between points of $R_{s}$ and $R_{\Phi}$ is thereby assured.* Figure 2 indicates the map.

* Cf. Osgood, W. F., Lehrbuch der Funktionentheorie, vol. 1, Leipzig, 1912, p. 377. 
The variables $\Phi$ and $\xi$ differ only by the real factor $\rho$, whence the domains $R_{\xi}$ and $R_{\Phi}$ differ only in scale. Figure 3 indicates the relation between $R_{\varepsilon}$

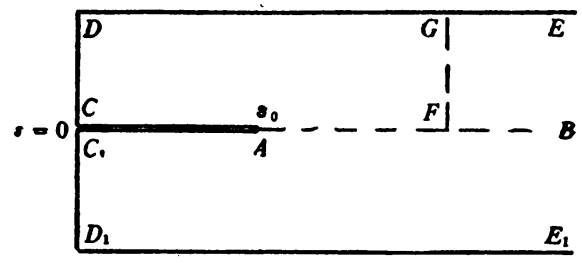

R.

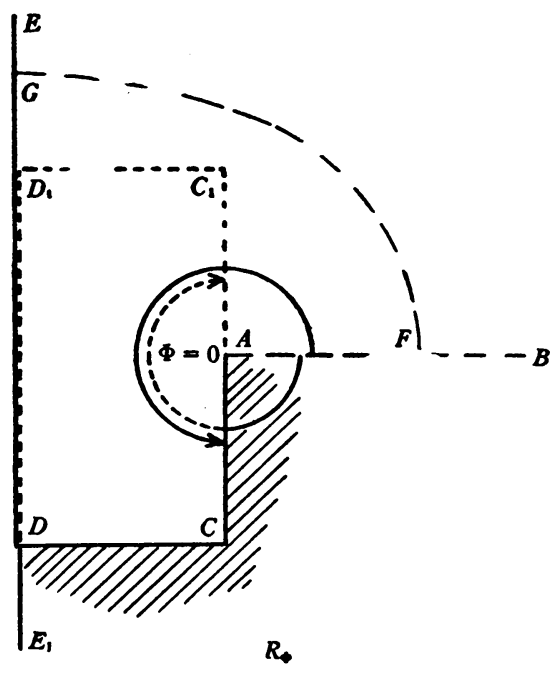

Fig. 2

and $R_{\xi}$, each domain being divided into the sub-regions $\Xi^{(l)}$ defined in (7). The lines by which this sub-division is effected need not be determined with

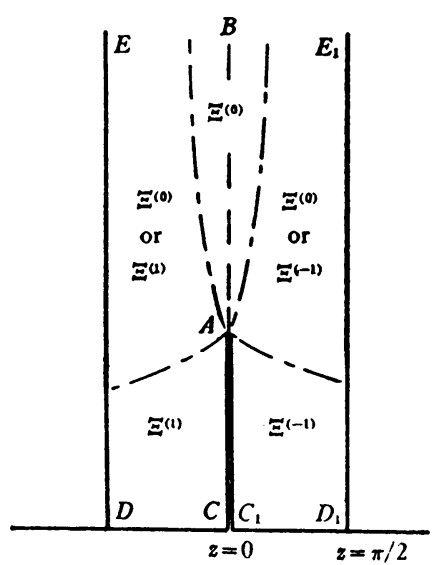

$R$,

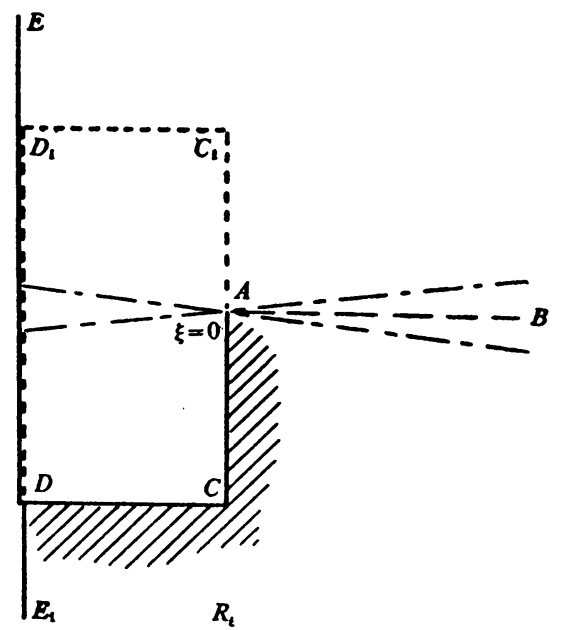

Fig. 3

precision, for due to the overlapping of the regions any displacement of the curves which does not affect the character of the figure is immaterial.

3.3. Fulfillment of the hypotheses. The zero of $\chi_{0}{ }^{2}(s)$ at $s_{0}^{\prime}$ is of the first order. Hence in the hypotheses of $\$ 1.2$ the values $\nu=1, \eta \equiv \omega_{1} \equiv k=0$ are to 
be used. With the value of $\phi$ given by the formula (3.3) it is found that the functions (6) are in the present instance

$$
\omega(\phi)=\frac{1}{4}\left\{-\frac{5}{9}\left(\frac{\phi}{\Phi}\right)^{2}+\sigma^{2}+\frac{6}{\phi^{2}}+\frac{5\left(2-\sigma^{2}\right)}{\phi^{4}}\right\}, \quad \Psi=\Phi^{1 / 6} / \phi^{1 / 2} .
$$

Let the region $R_{8}$.be divided into three parts by the relations

$$
\begin{aligned}
& \left|s-s_{0}^{\prime}\right| \leqq \delta, \\
\delta \leqq & \left|s-s_{0}^{\prime}\right| \leqq H, \\
H \leqq & \left|s-s_{0}^{\prime}\right|,
\end{aligned}
$$

with the constants $\delta$ and $H$ as specified in the following. It is to be shown that in each of these parts the hypotheses of $\$ 1.2$ are uniformly fulfilled.

To begin with let $H$ be chosen so large that in the part (c) the formulas (3.8) may be applied. Then it is a matter of simple computation to show that in this part of $R_{s}$ the hypotheses (i) and (iii) are uniformly fulfilled.

Next let $\delta$ be chosen so small that within the part (a), $\left|\phi^{2}\right| \leqq \frac{1}{2}$ for all admitted values of $\sigma$. Then

$$
\begin{aligned}
\left\{1+\frac{\sigma^{2} \phi^{2}}{2-\sigma^{2}}\right\}^{-1 / 2} & \equiv 1-\frac{\sigma^{2} \phi^{2}}{2\left(2-\sigma^{2}\right)}+\phi^{4} O(1), \\
\left(1+\phi^{2}\right)^{-1 / 2} & \equiv 1-\frac{\phi^{2}}{2}+\phi^{4} O(1),
\end{aligned}
$$

with $O(1)$ designating functions which are uniformly bounded. Since

$$
\Phi=\int_{0}^{\phi} \frac{\phi}{\phi^{\prime}} d \phi
$$

whereas from the formula (3.3)

$$
\frac{\phi}{\phi^{\prime}}=\frac{\phi^{2}}{\left(2-\sigma^{2}\right)^{1 / 2}}\left\{\left(1+\phi^{2}\right)\left(1+\frac{\sigma^{2} \phi^{2}}{2-\sigma^{2}}\right)\right\}^{-1 / 2},
$$

it is found that

$$
\Phi=\frac{\phi^{3}}{3\left(2-\sigma^{2}\right)^{1 / 2}}\left\{1-\frac{3 \phi^{2}}{5\left(2-\sigma^{2}\right)}+\phi^{4} O(1)\right\} .
$$

With this evaluation it is seen directly that in the part (a) the functions (3.9) are uniformly bounded.

Lastly in the part (b) the formula (3.3) may be written

$$
\chi_{0}^{2}(s)=2\left\{\left(2-\sigma^{2}\right)^{1 / 2} \frac{\sinh 2 \sigma\left(s-s_{0}^{\prime}\right)}{2 \sigma}+\frac{\sinh ^{2} \sigma\left(s-s_{0}^{\prime}\right)}{\sigma^{2}}\right\} .
$$


It is evident from this that both

$$
\chi_{0}(s) \text { and } \int_{s 0^{\prime}}^{s} \chi_{0}(s) d s
$$

are non-vanishing and continuous as functions of the two variables $\left(s-s_{0}^{\prime}, \sigma\right)$ in the closed region determined by (b) and (3.4). Accordingly, they are bounded uniformly in $\sigma$ and the hypothesis (i) is uniformly fulfilled. Clearly also the functions (3.9) are uniformly bounded and so the requirements of $\$ 1.2$ upon the differential equation are uniformly met.

3.4. The forms of the solutions. Since $\phi^{2}(s)$ has a simple zero in $R_{s}$ the asymptotic representation of any solution of the differential equation is subject to the Stokes' phenomenon, and $\nu$ being 1 the formulas of $\S 1.4$ are applicable. From Figure 3 it is seen that the origin $z=0$ may be regarded as lying in the sub-region $\Xi^{(-1)}$. Hence with $h=-1$ and the subscript $a$ replaced by 1 the formulas (11a) and (11b) yield the representations of the solutions $u_{o}(z)$ and $u_{e}(z)$. It may be observed from Figure 3, however, that the value $\xi_{1}$ which corresponds to $z=0$ (at $C_{1}$ in the figure) is such that $i \xi_{1}$ is real and negative, so that any quantity multiplied by $e^{i \xi_{1}}$ is asymptotically negligible in comparison with the same multiplied by $e^{-i \xi_{1}}$. With the omission of such negligible terms the formulas obtained are the following;

When $z$ is in $\Xi^{(l)}$, and $|\xi| \geqq N$,

$$
\begin{aligned}
& u_{o}(z)=\frac{1}{2}\left(\frac{\sigma^{2}}{\rho^{2} \phi_{1} \phi}\right)^{1 / 2}\left\{K_{0,1}^{-1 ; l} e^{i \xi}+K_{0,2}^{-1, l} e^{-i \xi}\right\}, \\
& u_{e}(z)=\frac{1}{2}\left(\frac{\phi_{1}}{\phi}\right)^{1 / 2}\left\{K_{e, 1}^{-1, l} e^{i \xi}+K_{e, 2}^{-1, l} e^{-i \xi}\right\},
\end{aligned}
$$

\begin{tabular}{|c|c|c|c|}
\hline$\therefore$ & -1 & 0 & 1 \\
\hline$K_{0,1}^{-1, l}$ & $e^{-1 k_{1}[1]}$ & $e^{-i \xi_{i}[1]}$ & $-i e^{i \xi_{i}[1]}$ \\
\hline$K_{0,2}^{-1, i}$ & $-e^{i \xi_{1}[1]}$ & $i e^{-i k_{1}[1]}$ & $i e^{-i \xi_{1}[1]}$ \\
\hline$K_{e, 1}^{-1, l}$ & $e^{-i \xi_{3}}[1]$ & $\because e^{-i \xi_{1}[1]}$ & $i e^{i \xi_{3}}[1]$ \\
\hline $\boldsymbol{K}_{i, 2}^{-1, t}$ & $e^{i \xi_{1}[1]}$ & $i e^{T i \xi_{1}}[1]$ & $i \sigma^{i \xi_{1}}[1]$ \\
\hline
\end{tabular}

with coefficients

(3.10a) 
When $|\xi| \leqq N$,

$$
u_{o}(z)=\left(\frac{\pi i \sigma^{2}}{6 \rho^{2} \phi_{1} \phi}\right)^{1 / 2} \xi^{1 / 6} e^{-i \xi_{1}}\left[\xi^{1 / 3} J_{-1 / 3}(\xi)+\xi^{1 / 3} J_{1 / 3}(\xi)\right],
$$

$$
u_{e}(z)=\left(\frac{\pi i \phi_{1}}{6 \phi}\right)^{1 / 2} \xi^{1 / 6} e^{-i \xi_{1}}\left[\xi^{1 / 3} J_{-1 / 3}(\xi)+\xi^{1 / 3} J_{1 / 3}(\xi)\right] .
$$

In the original variables

$$
\begin{aligned}
\frac{\rho \phi}{\sigma} & =\{\Omega \cos 2 z-\Delta\}^{1 / 2}, & \frac{\rho \phi_{1}}{\sigma} & =e^{-\pi i / 2}\{\Delta-\Omega\}^{1 / 2}, \\
\xi & =-i \int_{\nu_{0}}^{z}\{\Omega \cos 2 z-\Delta\}^{1 / 2} d z, & \xi_{1} & =i \int_{0}^{\nu_{0}}\{\Delta-\Omega \cosh 2 y\}^{1 / 2} d y,
\end{aligned}
$$

with $y_{0}=\frac{1}{2} \cosh ^{-1} \Delta / \Omega$. Further, it may be noted that since the values of $\phi$ on the lines $A C$ and $A C_{1}$ in Figure 3 differ only in sign, therefore

$$
\xi_{1}=\frac{-i \rho}{\sigma} \int_{A}^{C_{1}} \phi d z=\frac{i \rho}{\sigma} \int_{A}^{c} \phi d z
$$

whence the formulas

$$
\int_{0}^{z}\{\Omega \cos 2 z-\Delta\}^{1 / 2} d z=\left\{\begin{array}{l}
i\left(\xi-\xi_{1}\right), \text { in } \Xi^{(-1)}, \\
i\left(\xi+\xi_{1}\right), \text { in } \Xi^{(1)}
\end{array}\right.
$$

are also valid provided the entire path of integration is taken in each case in the sub-region indicated.

The formulas (11a), (11b) may likewise be drawn upon to give the representations of the solutions $u_{\alpha}(z), u_{\beta}(z)$. If the point corresponding to $z=\pi / 2$-is $s_{2}$, the subscript $a$ is to be replaced by 2 , and since $\xi_{2}$ (at $D_{1}$ in Figure 3 ) lies in the region $\Xi^{(-1)}, h$ is again to be taken as -1 . With the omission of asymptotically negligible terms the formulas obtained are the following:

When $z$ is in $\Xi^{(l)}$, and $|\xi| \geqq N$,

$$
u_{\alpha}(z)=\frac{1}{2}\left(\frac{\sigma^{2}}{\rho^{2} \phi_{2} \phi}\right)^{1 / 2}\left\{K_{\alpha, 1}^{-1, l} e^{i \xi}+K_{\alpha, 2}^{-1, l} e^{-i \xi}\right\}
$$

$$
u_{\beta}(z)=\frac{1}{2}\left(\frac{\phi_{2}}{\phi}\right)^{1 / 2}\left\{K_{\beta, 1}^{-1, l} e^{i \xi}+K_{\beta, 2}^{-1, l} e^{-i \xi}\right\}
$$

with coefficients 
(3.11a)

\begin{tabular}{|c|c|c|c|}
\hline & -1 & 0 & 1 \\
\hline$K_{\alpha, 1}^{-1, l}$ & $e^{-i \xi_{2}[1]}$ & $e^{-i \xi_{2}[1]}$ & $-i e^{i \xi_{2}[1]}$ \\
\hline$K_{\alpha, 2}^{-1, l}$ & $-e^{i \xi_{2}[1]}$ & $i e^{-i \xi_{2}[1]}$ & $i e^{-i \xi_{2}[1]}$ \\
\hline$K_{\beta, 1}^{-1, l}$ & $e^{-i \xi_{2}[1]}$ & $e^{-i \xi_{2}[1]}$ & $i e^{i \xi_{z}[1]}$ \\
\hline$K_{\beta, 2}^{-1, l}$ & $e^{i \xi_{2}[1]}$ & $i e^{-i \xi_{2}[1]}$ & $i e^{-i \xi_{2}[1]}$ \\
\hline
\end{tabular}

When $|\xi| \leqq N$,

$$
u_{\alpha}(z)=\left(\frac{\pi i \sigma^{2}}{6 \rho^{2} \phi_{2} \phi}\right)^{1 / 2} \xi^{1 / 6} e^{-i \xi_{2}}\left[\xi^{1 / 3} J_{-1 / 8}(\xi)+\xi^{1 / 3} J_{1 / 3}(\xi)\right],
$$

$$
u_{\beta}(z)=\left(\frac{\pi i \phi_{2}}{6 \phi}\right)^{1 / 2} \xi^{1 / 6} e^{-i \xi_{2}}\left[\xi^{1 / 3} J_{-1 / 3}(\xi)+\xi^{1 / 3} J_{1 / 8}(\xi)\right] .
$$

Again

$$
\begin{aligned}
\frac{\rho \phi_{2}}{\sigma} & =e^{-\pi i / 2}\{\Delta+\Omega\}^{1 / 2} \\
\xi_{2} & =\xi_{1}-\int_{0}^{\pi / 2}\{\Delta-\Omega \cos 2 x\}^{1 / 2} d x .
\end{aligned}
$$

Figure 3 shows that the segments $-\pi / 2 \leqq x \leqq 0$ and $0 \leqq x \leqq \pi / 2$ of the axis of reals lie respectively in the sub-regions $\Xi^{(1)}$ and $\Xi^{(-1)}$. The formulas above appropriate to these regions accordingly yield the descriptions of the solutions when $z$ is real. It is found that these formulas are precisely those given in (2.8b) and (2.9b), though it should be noted that with the difference in the definition of the parameter $\rho$ the significance of symbol [ ] is slightly different in this chapter from that in the preceding one.

The pairs of solutions (3.10) and (3.11) have each the defect that in the region about the upper part of the axis of imaginaries the component solutions are asymptotically multiples of each other. The pair of solutions $u_{-1,1}, u_{-1,2}$ given in (9) would be one not subject to this particular shortcoming.

3.5. The solutions of the associated Mathieu equation. If $z$ lies in any of the domains indicated in Figure 4, the point $i z$ lies in the corresponding 
sub-region of $R_{\mathrm{z}}$ as shown in Figure 3. In accordance with (12) the representations of $i v_{o}(z)$ and $v_{\odot}(z)$ are therefore obtainable in any one of the regions

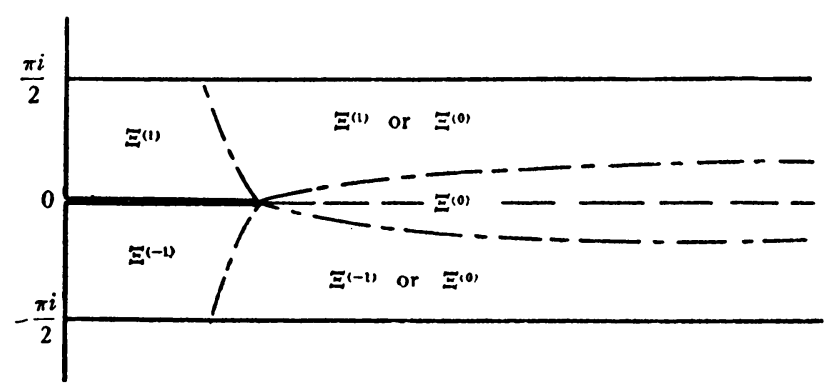

FIG. 4

indicated by the mere substitution in the associated formulas (3.10) of $\bar{\phi}$ and $\bar{\xi}$ in place of $\phi$ and $\xi$, the former being the same functions of $i z$ as the latter are of $z$. Explicitly

$$
\begin{aligned}
\frac{\rho \bar{\phi}}{\sigma} & =\{\Omega \cosh 2 z-\Delta\}^{1 / 2}, \\
\bar{\xi} & =\int_{x_{0}}^{z}\{\Omega \cosh 2 z-\Delta\}^{1 / 2} d z, \quad x_{0}=\frac{1}{2} \cosh ^{-1} \Delta / \Omega .
\end{aligned}
$$

In particular, for real values of the variable the formulas so obtained are the following:

For $0 \leqq x<x_{0},|\bar{\xi}| \geqq N$,

$$
v_{0}(x)=\frac{[1]}{\{(\Delta-\Omega)(\Delta-\Omega \cosh 2 x)\}^{1 / 4}} \sinh \left[\int_{0}^{x}\{\Delta-\Omega \cosh 2 x\}^{1 / 2} d x\right],
$$

$$
\nu_{e}(x)=\left\{\frac{\Delta-\Omega}{\Delta-\Omega \cosh 2 x}\right\}^{1 / 4}[1] \cosh \left[\int_{0}^{x}\{\Delta-\Omega \cosh 2 x\}^{1 / 2} d x\right] .
$$

For $x \leqq x_{0},|\bar{\xi}| \leqq N$,

(3.12b) $v_{o}(x)=\frac{(2 \pi)^{-1 / 2}|\bar{\xi}|^{1 / 6} e^{-i \xi_{1}}}{\{(\Delta-\Omega)(\Delta-\Omega \cosh 2 x)\}^{1 / 4}}\left[|\bar{\xi}|^{1 / 3} K_{1 / 3}(|\bar{\xi}|)\right]$.

For $x_{0} \leqq x,|\bar{\xi}| \leqq N$,

(3.12c) $v_{o}(x)=\frac{(\pi / 6)^{1 / 2} \bar{\xi}^{1 / 6} e^{-i \xi_{1}}}{\{(\Delta-\Omega)(\Omega \cosh 2 x-\Delta)\}^{1 / 4}}\left[\bar{\xi}^{1 / 3} J_{-1 / 3}(\bar{\xi})+\bar{\xi}^{1 / 3} J_{1 / 3}(\bar{\xi})\right]$. 
For $x<x_{0},|\bar{\xi}| \geqq N$,

(3.12d) $v_{o}(x)=\frac{[1] e^{-i \xi_{1}}}{\{(\Delta-\Omega)(\Omega \cosh 2 x-\Delta)\}^{1 / 4}} \cos \left[\int_{x_{0}}^{x}\{\Omega \cosh 2 x-\Delta\}^{1 / 2} d x-\frac{\pi}{4}\right]$.

For the $x$ ranges concerned in the cases (b), (c) and (d) the representation of $v_{e}(x)$ has been omitted since it is found to differ in appearance from that of $v_{0}(x)$ only in that the factor $(\Delta-\Omega)^{-1 / 4}$ is replaced by $(\Delta-\Omega)^{1 / 4}$. For the range in case (b) the value of $\bar{\xi}$ is imaginary, i.e., $\bar{\xi}=e^{-8 \pi i / 2}|\bar{\xi}|$, and the relation

$$
J_{-1 / 3}(\bar{\xi})+J_{1 / 3}(\bar{\xi})=\frac{3^{1 / 2} i}{\pi} K_{1 / 3}(|\bar{\xi}|)
$$

was used.

As already noted in $\$ 3.4$, a pair of solutions which unlike those above are not asymptotically multiples of each other for large real values of $z$ would be that obtainable in the manner used above from the functions $u_{-1, j}(z)$ described in (9).

3.6. The characteristic values and exponent. The forms of both the exponent $\mu$ and the characteristic equations were found in chapter 2 to be determined by the formulas (2.9b). Since these formulas, except for the interpretation of the symbol [ ], remain valid for the configuration at present under discussion, the deductions of $\$ 2.5$ and $\$ 2.4$ require but slight modification to apply to the case in hand. The characteristic exponent is thus given by the formula

$$
\mu=\frac{i}{\pi} \cos ^{-1}\left\{\cos \int_{0}^{\pi / 2} 2\{\Delta-\Omega \cos 2 x\}^{1 / 2} d x+O\left(\frac{\Delta^{1 / 2}}{\Delta-\Omega}\right)\right\} .
$$

The order of the final term within the bracket evidently increases with $\Omega$, from which it is evident that the domain of parameter values for which $\mu$ is real, i.e., for which there are unstable solutions, increases in extent as the upper end of the range of values $\Omega$ admitted in the configuration of the present chapter is approached.

The characteristic values $S_{n}(\Omega)$ and $C_{n}(\Omega)$ are each the root of an equation of the form (2.12) which in the present instance is more explicitly

$$
\int_{0}^{\pi / 2}\{\Delta-\Omega \cos 2 x\}^{1 / 2} d x+O\left(\frac{\Delta^{1 / 2}}{\Delta-\Omega}\right)=\frac{n \pi}{2} .
$$

The lower end of the $\Omega$ range joins with that of the configuration II, and for such parameter values the formulas (2.13) are again valid as was to be ex- 
pected. To obtain formulas valid near the upper end of the range the following process may be used.

Let $k_{1}$ be defined by the relation

$$
\Delta-\Omega=2^{5 / 2} k_{1} \Omega^{1 / 2},
$$

and in the integral of (3.14) replace $x$ by $\pi / 2-\zeta$. Then the equation becomes

$$
(\Delta+\Omega)^{1 / 2} G\left(h^{2}, h^{2}\right)+O\left(\frac{\Delta^{1 / 2}}{\Delta-\Omega}\right)=\frac{n \pi}{2},
$$

with $G$ the elliptic integral of (26) and

$$
h^{2}=\left(1+\frac{4 k_{1}}{\Omega}\right)^{-1} \text {. }
$$

For the larger of the admitted values of $\Omega$ the ratio $k_{1} / \Omega$ is of the order of $\Delta^{-1 / 2}$ and $h^{2}$ is therefore nearly 1 . With the use of the formula (26b) the equation may accordingly be written

$$
(2 \Omega)^{1 / 2}-k_{1} \log \frac{k_{1}}{(32 \Omega)^{1 / 2}}+k_{1}
$$

$$
+k_{1} O\left(\frac{k_{1}}{\Omega^{1 / 2}} \log \frac{k_{1}}{\Omega}\right)+O\left(\frac{\Delta^{1 / 2}}{\Delta-\Omega}\right)=\frac{n \pi}{2} .
$$

Recalling (3.15), therefore, it follows that

$$
\begin{aligned}
& S_{n}(\Omega)=\Omega+2^{5 / 2} k_{1}(n) \Omega^{1 / 2}, \\
& C_{n}(\Omega)=\Omega+2^{5 / 2} k_{1}(n) \Omega^{1 / 2},
\end{aligned}
$$

with each $k_{1}(n)$ a root of an equation of the form (3.14b).

Chapter 4

The CONFIgURATION IV

4.1. The differential equation. Let the configuration designated as IV in Figure 1 be defined as that comprising the parameter values $(\Omega, \Delta)$ in which both are large and

$$
-M_{2} \Omega^{1 / 2} \leqq \Delta-\Omega \leqq M_{2} \Delta^{1 / 2},
$$

$M_{2}$ being the constant in the relation (3.1). Then the substitutions

$$
\rho=(32 \Omega)^{1 / 2}, \quad \sigma=\frac{\Delta-\Omega}{(32 \Omega)^{1 / 2}}, \quad s=z
$$

determine $\rho$ as a large parameter, while the range of values given to $\sigma$ is 
bounded. The differential equation (1) takes the form (3) with the coefficients

$$
\begin{aligned}
& \chi_{0} \equiv \frac{1}{2} \sin s, \\
& \chi_{1} \equiv \sigma,
\end{aligned}
$$

in virtue of which the functions (5) are in this case explicitly

$$
\begin{aligned}
k & =-i \sigma, \\
\eta(s) & =2 i \sigma \tan \frac{s}{2}, \\
\phi & =\sin \frac{s}{2}\left\{\frac{1}{2} \cos \frac{s}{2}+\frac{\sigma}{\rho} \sec \frac{s}{2}\right\}, \\
\Phi & =\frac{1}{2} \sin ^{2} \frac{s}{2}-\frac{\sigma}{\rho} \log \cos ^{2} \frac{s}{2} .
\end{aligned}
$$

Let $R_{z}$ be chosen as the strip (13). Then in the region $R_{s}$ the coefficient $\chi_{0}{ }^{2}$ has a single zero located at the origin and of the second order. It must be shown that with the appropriate values $s_{0}=0, \nu=2$ the requirements of $\$ 1.2$ are uniformly fulfilled. The hypotheses (i) and (ii) offer no difficulty in this respect, while the consideration of the functions (6) and the hypothesis (iii) may be made as follows.

The relation

$$
e^{q}=\cos ^{2} \frac{s}{2}
$$

defines $q$, in terms of which

$$
\begin{aligned}
& \Phi=q e^{q}\left\{\frac{e^{-q}-1}{2 q}-\frac{\sigma}{\rho} e^{-q}\right\}, \\
& \omega_{1}=\sigma^{2}\left\{1-e^{-q}+\frac{2 e^{q}-2-q e^{q}+\frac{2 \sigma}{\rho}\left(1-e^{-q}\right)}{\Phi}\right\}
\end{aligned}
$$

while the various members of the formula

$$
\omega(\phi)=\frac{3}{16}\left(\frac{2 \phi^{\prime}}{\phi}-\frac{\phi}{\Phi}\right)\left(\frac{2 \phi^{\prime}}{\phi}+\frac{\phi}{\Phi}\right)-\frac{\phi^{\prime \prime}}{2 \phi}
$$

are found to be 


$$
\begin{aligned}
\frac{\phi^{\prime \prime}}{\phi} & =-\frac{1+\frac{\sigma}{\rho} e^{-2 q}}{1+\frac{\sigma}{\rho} e^{-q}}, \\
\frac{2 \phi^{\prime}}{\phi} & =\cot \frac{s}{2}-\tan \frac{s}{2}\left\{\frac{1-\frac{2 \sigma}{\rho} e^{-q}}{1+\frac{2 \sigma}{\rho} e^{-q}}\right\}, \\
\frac{\phi}{\Phi} & =\cot \frac{s}{2}-\tan \frac{s}{2}\left\{\frac{2 \sigma\left(q-1+e^{-q}\right)}{\rho \Phi\left(e^{-q}-1\right)}\right\} .
\end{aligned}
$$

It is to be observed now that $q$ vanishes with $s$, that $\left|e^{q}\right| \geqq \frac{1}{2}$ in $R_{s}$, and that the ratio $\sigma / \rho$ will be uniformly as small as desired if $\Omega$ is restricted to remain sufficiently large. It is consequently seen that the brace in the formula for $\Phi$ is uniformly bounded from zero and hence that both $\omega(\phi)$ and $\omega_{1}$ are uniformly bounded in any finite part of $R_{\mathbf{s}}$. Finally, when $|s|$ is great the asymptotic formulas

$$
\begin{aligned}
\phi & \sim \frac{ \pm i}{2} e^{q}, & \omega(\phi) & \sim \frac{-1}{16}, \\
\omega_{1} & \sim 2 \sigma^{2} q, & d s & \sim \pm i d q
\end{aligned}
$$

are readily checked and in virtue of them the uniform fulfillment of the hypothesis (iii) becomes evident.

4.2. The solutions $u_{o}(z)$ and $u_{e}(z)$. The variables $\Phi$ and $\xi$ differ only by the real factor $\rho$, while $s$ and $z$ are identical. Since the values of $\Phi$ on the boundaries of $R_{\mathrm{s}}$ are as follows:

for $s^{\prime}=0$,

$$
\Phi=-\frac{1}{2} \sinh ^{2} \frac{s^{\prime \prime}}{2}-\frac{\sigma}{\rho} \cdot \log \cosh ^{2} \frac{s^{\prime \prime}}{2}
$$

for $s^{\prime}=\pi / 2$,

$$
\Phi=\left\{\frac{1}{4}-\frac{\sigma}{\rho} \log \frac{\cosh s^{\prime \prime}}{2}\right\}+i\left\{\frac{\sinh s^{\prime \prime}}{4}+\frac{\sigma}{\rho} \tan ^{-1}\left(\sinh s^{\prime \prime}\right)\right\},
$$

the map of $R_{z}$ upon $R_{\xi}$ is as indicated in Figure 5. The figure shows also the partition of these regions into the sub-regions $\Xi^{(l)}$ defined in (7). 
The representation of a pair of solutions $u_{1}(s), u_{2}(s)$ which are determined by the initial values

$$
\begin{array}{ll}
u_{1}(0)=0, & u_{1}^{\prime}(0)=\left(\frac{i \rho}{4}\right)^{1 / 2}\left(1+\frac{2 \sigma}{\rho}\right)^{1 / 4}, \\
u_{2}(0)=\left(1+\frac{2 \dot{\sigma}}{\rho}\right)^{-1 / 4}, & u_{2}^{\prime}(0)=0
\end{array}
$$

is known, ${ }^{*}$ and is expressible in terms of the confluent hypergeometric func-
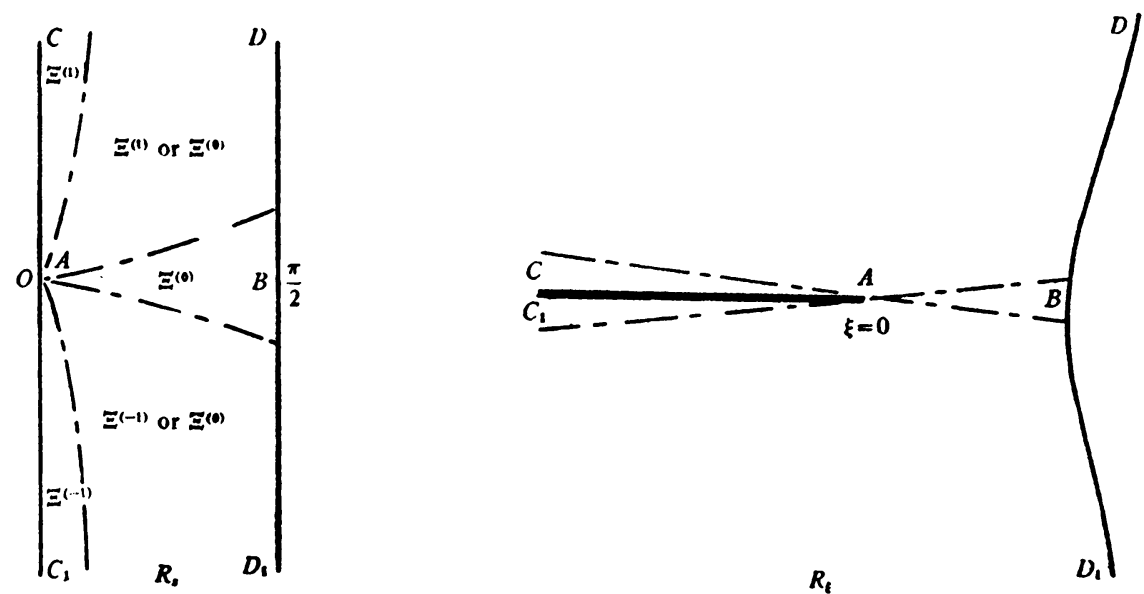

Fig. 5

tions customarily designated by $M_{j, l} . \dagger$ With the functions $\mathcal{X}_{j}$ defined by the formulas

$$
\begin{aligned}
& \mathcal{X}_{1}(\xi, \sigma)=e^{-8 x i / 8 \xi-1 / 4} \mathcal{X}_{-i \sigma, 1 / 4}(2 i \xi), \\
& \mathcal{X}_{2}(\xi, \sigma)=e^{-\pi i / 8 \xi^{-1 / 4}} \mathcal{X}_{-i \sigma,-1 / 4}(2 i \xi),
\end{aligned}
$$

it is found thus that the principal solutions, which are evidently mere multiples of $u_{1}$ and $u_{2}$, are the following:

For $|\xi| \leqq N$,

$$
\begin{aligned}
& u_{o}(z)=\left(\frac{2}{\rho}\right)^{1 / 2} \Psi\left[M_{1}(\xi, \sigma)\right], \\
& u_{\theta}(z)=\left(\frac{1}{2}\right)^{1 / 2} \Psi\left[M_{2}(\xi, \sigma)\right] .
\end{aligned}
$$

* Paper $\mathrm{L}_{3}$. See, however, the footnote on $\mathrm{p} .646$ regarding the differences of notation.

† Cf. Whittaker and Watson, loc. cit., chapter XVI. 
On the other hand, when $z$ is not in the neighborhood of the origin the formulas are the following*:

For $|\xi| \geqq N$, and $z$ in $\Xi^{(l)}$,

$$
\begin{aligned}
u_{o}(z)= & \left(\frac{\pi}{2 \phi}\right)^{1 / 2}\left(i_{\rho}\right)^{-3 / 4}\left\{\left[\frac{k_{0,1}^{(l)}}{\Gamma\left(\frac{3}{4}+i \sigma\right)}\right]_{1}(2 i \xi)^{i \sigma} e^{i \xi}\right. \\
& \left.+\left[\frac{k_{0,2}^{(l)}}{\Gamma\left(\frac{3}{4}-i \sigma\right)}\right]_{1}(2 i \xi)^{-i \sigma} e^{-i \xi}\right\},
\end{aligned}
$$

with coefficients

$$
\begin{aligned}
u_{e}(z)= & \left(\frac{\pi}{2 \phi}\right)^{1 / 2}(i \rho)^{-1 / 4}\left\{\left[\frac{k_{e, 1}^{(l)}}{\Gamma\left(\frac{1}{4}+i \sigma\right)}\right]_{1}(2 i \xi)^{i \sigma} e^{i \xi}\right. \\
& \left.+\left[\frac{k_{e, 2}^{(l)}}{\Gamma\left(\frac{1}{4}-i \sigma\right)}\right]_{1}(2 i \xi)^{-i \sigma} e^{-i \xi}\right\},
\end{aligned}
$$

\begin{tabular}{|c|c|c|c|}
\hline$l$ & -1 & 0 & 1 \\
\hline$k_{0,1}^{(l)}$ & 1 & 1 & $-i e^{2 \sigma x}$ \\
\hline$k_{0,2}^{(l)}$ & $e^{\sigma \pi-3 \pi i / 4}$ & $e^{-\sigma \pi+3 \pi i / 4}$ & $e^{-\sigma \pi+3 \pi i / 4}$ \\
\hline$k_{e, 1}^{(l)}$ & 1 & 1 & $i e^{2 \sigma \pi}$ \\
\hline$k_{e, 2}^{(l)}$ & $e^{\sigma \pi-\pi i / 4}$ & $e^{-\sigma \pi+\pi i / 4}$ & $e^{-\sigma \pi+\pi i / 4}$ \\
\hline
\end{tabular}

$(4.6 c)$

for use in these formulas it is permissible to write in terms of the original variables

$$
\begin{aligned}
\phi & =\left[\frac{1}{4}\right] \sin z, \quad \xi=(2 \Omega)^{1 / 2}[1](1-\cos z), \\
e^{i \xi} & =\left(\frac{2}{1+\cos z}\right)^{i \sigma} e^{(i \rho / 4)(1-\cos z)}, \Psi=\left(\frac{1}{1+\cos z}\right)^{1 / 4}[1] .
\end{aligned}
$$

When $z$ is real the same is true of $\phi, \Psi$ and $\xi$, and the last of these is positive. For such values the functions $\mathscr{X}_{i}$ of (4.5) are real, and the formulas (4.6a) are therefore directly real. From Figure 5 it is seen that such values of $z$ lie in $\Xi^{(0)}$, whence the appropriate formulas $(4.6 \mathrm{~b})$ reduce to

* The symbol []$_{1}$ is used in the sense that $[Q]_{1}$ denotes a quantity which differs from $Q$ by terms of the order of $(\log \rho) / \rho$ and terms of the order of $N^{-1}$. 
$(4.6 \mathrm{~d})$

$$
\begin{aligned}
& u_{o}(x)=\rho^{-3 / 4}\left(\frac{2 \pi}{\phi}\right)^{1 / 2} e^{-\sigma \pi / 2}\left[\frac{1}{\Gamma_{1}}\right]_{1} \sin \left[\xi+\sigma \log 2 \xi-\gamma_{1}+\frac{\pi}{8}\right]_{1}, \\
& u_{e}(x)=\rho^{-1 / 4}\left(\frac{2 \pi}{\phi}\right)^{1 / 2} e^{-\sigma \pi / 2}\left[\frac{1}{\Gamma_{2}}\right]_{1} \cos \left[\xi+\sigma \log 2 \xi-\gamma_{2}-\frac{\pi}{8}\right]_{1} .
\end{aligned}
$$

The symbols $\Gamma_{j}$ and $\gamma_{j}$ designate the real values determined by the formulas

$$
\Gamma\left(\frac{3}{4} \pm i \sigma\right)=\Gamma_{1} e^{ \pm i \gamma_{1}}, \quad \Gamma\left(\frac{1}{4} \pm i \sigma\right)=\Gamma_{2} e^{ \pm i \gamma_{2}},
$$

in which the left-hand members are gamma functions.

4.3. The solutions $u_{\alpha}(z)$ and $u_{\beta}(z)$. The solutions of the equation (3) especially associated with the sub-region $\Xi^{(0)}$ which by Figure 5 contains the point $z=\pi / 2$, are those described by the following formulas:

For $|\xi| \geqq N$, and $s$ in $\Xi^{(l)}$,

$$
u_{0, j}(s)=(i \rho)^{-1 / 4}(2 \phi)^{-1 / 2}\left\{B_{j, 1}^{(l)}(2 i \xi)^{i \sigma} e^{i \xi}+B_{i, 2}^{(l)}(2 i \xi)^{-i \sigma} e^{-i \xi}\right\},
$$

with coefficients

$(4.9 b)$

\begin{tabular}{c|c|c|c|}
\hline & -1 & 0 & 1 \\
\hline$B_{1,1}^{(l)}$ & {$[1]_{1}$} & {$[1]_{1}$} & {$[1]_{1}$} \\
\hline$B_{1,2}^{(l)}$ & {$\left[\frac{-2 \pi i}{\Gamma\left(\frac{3}{4}-i \sigma\right) \Gamma\left(\frac{1}{4}-i \sigma\right)}\right]_{1}$} & 0 & 0 \\
\hline$B_{2,1}^{(l)}$ & 0 & 0 & {$\left[\frac{2 \pi i e^{2 \sigma \pi}}{\Gamma\left(\frac{3}{4}+i \sigma\right) \Gamma\left(\frac{1}{4}+i \sigma\right)}\right]_{1}$} \\
\hline$B_{2,2}^{(l)}$ & {$[1]_{1}$} & {$[1]_{1}$} & {$[1]_{1}$} \\
\hline
\end{tabular}

For $|\xi| \leqq N$,

$$
\begin{aligned}
u_{0,1}= & i\left(\frac{\pi}{2}\right)^{1 / 2} e^{-\sigma \pi} \Psi\left\{\frac{2}{\Gamma\left(\frac{1}{4}-i \sigma\right)}\left[\mathcal{H}_{1}(\xi, \sigma)\right]_{1}\right. \\
& \left.-\frac{e^{\pi i / 4}}{\Gamma\left(\frac{3}{4}-i \sigma\right)}\left[\mathcal{X}_{2}(\xi, \sigma)\right]_{1}\right\} \\
u_{0,2}= & -\left(\frac{\pi}{2}\right)^{1 / 2} \Psi\left\{\frac{2 e^{\pi i / 4}}{\Gamma\left(\frac{1}{4}+i \sigma\right)}\left[\mathcal{X}_{1}(\xi, \sigma)\right]_{1}\right. \\
& \left.-\frac{1}{\Gamma\left(\frac{3}{4}+i \sigma\right)}\left[\mathcal{X}_{2}(\xi, \sigma)\right]_{1}\right\} .
\end{aligned}
$$


The substitution into the formulas (8b) is simple, the Wronskian having the value $W=\left(i_{\rho}\right)^{1 / 2}[1]_{1}$, and if the subscript 2 is used to designate evaluations at $z=\pi / 2$, it is thus found that we have the following:

For $|\xi| \geqq N$, and $z$ in $\Xi^{(l)}$

$$
\begin{aligned}
u_{\alpha}(z)= & \frac{1}{2 i \rho \phi_{2}{ }^{1 / 2} \phi^{1 / 2}}\left\{\left(\frac{\xi}{\xi_{2}}\right)^{i \sigma} e^{i\left(\xi-\xi_{2}\right)}\left[1-\theta_{1}\right]_{1}\right. \\
& \left.-\left(\frac{\xi}{\xi_{2}}\right)^{-i \sigma} e^{-i\left(\xi-\xi_{2}\right)}\left[1-\theta_{2}\right]_{1}\right\} \\
u_{\beta}(z)= & \frac{\phi_{2}{ }^{1 / 2}}{2 \phi^{1 / 2}}\left\{\left(\frac{\xi}{\xi_{2}}\right)^{i \sigma} e^{i\left(\xi-\xi_{2}\right)}\left[1+\theta_{1}\right]_{1}\right. \\
& \left.+\left(\frac{\xi}{\xi_{2}}\right)^{-i \sigma} e^{-i\left(\xi-\xi_{2}\right)}\left[1+\theta_{2}\right]_{1}\right\}
\end{aligned}
$$

where$$
\theta_{1}=B_{2,1}^{(l)}\left(-4 \xi_{2}^{2}\right)^{i \sigma} e^{2 i \xi_{2}},
$$$$
\theta_{2}=B_{1,2}^{(l)}\left(-4 \xi_{2}^{2}\right)^{-i \sigma} e^{-2 i \xi_{2}} \text {. }
$$

For $|\xi| \leqq N$,

$$
u_{\alpha}(z)=2 \pi^{1 / 2} \rho^{-3 / 4} e^{-\sigma \pi / 2} \Psi\left\{\frac{2 \cos \varepsilon_{2}}{\Gamma_{2}}\left[\mathscr{X}_{1}(\xi, \sigma)\right]_{1}-\frac{\sin \varepsilon_{1}}{\Gamma_{1}}\left[\mathcal{X}_{2}(\xi, \sigma)\right]_{1}\right\},
$$

$$
u_{\beta}(z)=\pi^{1 / 2} \rho^{1 / 4} e^{-\sigma \pi / 2} \Psi\left\{\frac{\sin \varepsilon_{2}}{\Gamma_{2}}\left[\mathscr{x}_{1}(\xi, \sigma)\right]_{1}+\frac{\cos \varepsilon_{1}}{\Gamma_{1}}\left[\mathscr{H}_{2}(\xi, \sigma)\right]_{1}\right\},
$$

with

$$
\begin{aligned}
& \varepsilon_{1}=\left[\frac{\rho}{4}+\sigma \log \rho-\gamma_{1}+\frac{\pi}{8}\right]_{1}, \\
& \varepsilon_{2}=\left[\frac{\rho}{4}+\sigma \log \rho-\gamma_{2}-\frac{\pi}{8}\right]_{1} .
\end{aligned}
$$

For real values of $z$ the formulas $(4.10 \mathrm{~b})$ are directly real, while the appropriate formulas from $(4.10 \mathrm{a})$ reduce to

$$
\begin{aligned}
& u_{\alpha}(x)=\frac{-[2]_{1}}{\rho \phi^{1 / 2}} \sin \left[\frac{\rho}{4} \cos x-2 \sigma \log \tan \frac{x}{2}\right]_{1}, \\
& u_{\beta}(x)=\frac{[1]_{1}}{2 \phi^{1 / 2}} \cos \left[\frac{\rho}{4} \cos x-2 \sigma \log \tan \frac{x}{2}\right]_{1} .
\end{aligned}
$$

4.4. The solutions of the associated Mathieu equation. The representation of the solutions $i v_{o}(z)$ and $v_{e}(z)$, of the "associated" differential equation 
(2), are obtainable, as is now familiar, from the formulas of $\$ 4.2$ by the substitution in place of $\phi, \xi$ and $\Psi$ of the respective functions of $i z$, which may be designated by $\bar{\phi}, \bar{\xi}$ and $\bar{\Psi}$. Explicitly the evaluations

$$
\begin{aligned}
\bar{\phi} & =\left[\frac{i}{4}\right] \sinh z \\
\bar{\xi} & =(2 \Omega)^{1 / 2}[1](1-\cosh z), \\
e^{i \bar{\xi}} & =\left(\frac{2}{1+\cosh z}\right)^{i \sigma} e^{(i \rho / 4)(1-\cosh z)} \\
\bar{\Psi} & =\left(\frac{4}{1+\cosh z}\right)^{1 / 4}[1]
\end{aligned}
$$

may be used. The sub-regions of the $z$ plane in which the respective formulas so derived are valid are as is shown in Figure 6.

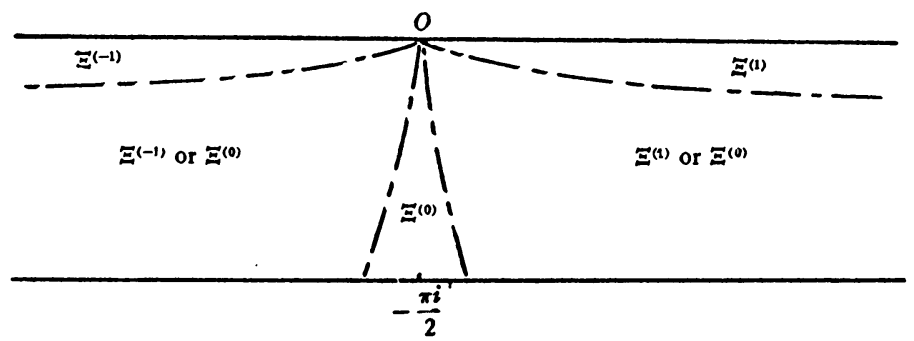

FIG. 6

In particular, when $z$ is real and positive the forms deduced from (4.6b) for $\Xi^{(1)}$ reduce to the following:

for $|\bar{\xi}| \geqq N$,

$$
v_{o}(x)=\rho^{-3 / 4}\left(\frac{2 \pi}{|\bar{\phi}|}\right)^{1 / 2} e^{\sigma \pi / 2}\left[\frac{1}{\Gamma_{1}}\right]_{1} \sin \left[|\bar{\xi}|-\sigma \log 2|\bar{\xi}|+\gamma_{1}+\frac{\pi}{8}\right]_{1},
$$

$$
v_{e}(x)=\rho^{-1 / 4}\left(\frac{2 \pi}{|\bar{\phi}|}\right)^{1 / 2} e^{\sigma \pi / 2}\left[\frac{1}{\Gamma_{2}}\right]_{1} \cos \left[|\bar{\xi}|-\sigma \log 2|\bar{\xi}|+\gamma_{2}-\frac{\pi}{8}\right]_{1}
$$

On the other hand, when $x$ is small, i.e., for $|\bar{\xi}| \leqq N$,

$$
\begin{aligned}
& v_{o}(x)=\left(\frac{2}{\rho}\right)^{1 / 2} \Psi\left[\mathcal{I X}_{1}(-|\bar{\xi}|, \sigma)\right], \\
& v_{e}(x)=\left(\frac{1}{2}\right)^{1 / 2} \Psi\left[\mathcal{X}_{2}(-|\bar{\xi}|, \sigma)\right] .
\end{aligned}
$$


The functions within the brackets may be shown to be explicitly real as they should be.

4.5. The characteristic values. The values (4.6d) substituted into the characteristic equations (17) and (18) give to the latter the forms

$$
(2 \Omega)^{1 / 2}+\frac{\sigma}{2} \log (32 \Omega)-\gamma_{1}+\frac{\pi}{8}+O\left(\Omega^{-1 / 2} \log \Omega\right)=\frac{n \pi}{2},
$$

for an odd Mathieu function,

$$
(2 \Omega)^{1 / 2}+\frac{\sigma}{2} \log (32 \Omega)-\gamma_{2}-\frac{\pi}{8}+O\left(\Omega^{-1 / 2} \log \Omega\right)=\frac{n \pi}{2},
$$

for an even Mathieu function.

These equations may be given a somewhat more detailed form when $\sigma$ is near either the one or the other extreme or the middle of its admitted range of values. The indices of the characteristic values which satisfy the equations with a specific integer $n$ on the right may also be determined as will be shown.

The theory of the gamma function supplies, in particular when $c_{1}=3 / 4$ and $c_{2}=1 / 4$, the formulas*

$$
\gamma_{j}=\sigma \frac{\Gamma^{\prime}\left(c_{j}\right)}{\Gamma\left(c_{j}\right)}+\sum_{r=1}^{\infty}\left(\frac{\sigma}{c_{j}+r}-\tan ^{-1} \frac{\sigma}{c_{j}+r}\right)
$$

$$
\begin{aligned}
\log \Gamma\left(c_{j}+i \sigma\right)= & \frac{1}{2} \log 2 \pi+\left(c_{j}-\frac{1}{2}+i \sigma\right) \log \left(c_{i}+i \sigma\right) \\
& -\left(c_{j}+i \sigma\right)+O\left(\frac{1}{|\sigma|}\right), \\
\Gamma(\zeta) \Gamma(1-\zeta)= & \pi \csc \pi \zeta,
\end{aligned}
$$

and from the first of these it is readily seen that with $\Omega$ fixed the left members of the equations (4.13) vary monotonically with $\sigma$ so that the roots for any integer $n$ are unique.

When $\sigma$ is near the upper end of its admitted range of values, it is large and positive, and the second of the formulas (4.14) gives the evaluations

$$
\gamma_{j}=\sigma \log \sigma-\sigma+\left(2 c_{i}-1\right)(\pi / 4)+O(1 / \sigma) .
$$

Both the equations (4.13) thus become

(4.13a) $(2 \Omega)^{1 / 2}-\frac{\sigma}{2} \log \frac{\sigma^{2}}{32 \Omega}+\sigma+O\left(\Omega^{1 / 2} \log \Omega\right)+O\left(\frac{\Omega^{1 / 2}}{\Delta-\Omega}\right)=\frac{n \pi}{2}$,

* Cf. Nielsen, N., Handbuch der Theorie der Gammafunktion, Leipzig, 1906, p. 23 and pp. 94 and 209. 
which is, therefore, the form of the characteristic equations when $\Omega$ is near the lower end of the range of values admitted for it in the present configuration. Since for these values the configurations of the present and the preceding chapter abut, the indices of the characteristic values concerned may be determined by a comparison of the equations (4.13a) and (3.16), $k_{1}$ in the latter having been defined precisely as $\sigma$ is in the former. With a given value of $n$ the roots of the equations (4.13) are thus seen to be precisely $S_{n}(\Omega)$ and $C_{n}(\Omega)$ respectively.

Near the middle of its range $\sigma$ is small, and the left members of the equations (4.13) are essentially represented by the early terms of their expansions in powers of $\sigma$. Thus the equations become

$$
\begin{aligned}
\left\{\left(n+\frac{1}{2}-c_{j}\right)\right. & \left.\frac{\pi}{2}-(2 \Omega)^{1 / 2}+O\left(\Omega^{-1 / 2} \log \Omega\right)\right\} \\
& +\sigma\left\{\frac{\Gamma^{\prime}\left(c_{j}\right)}{\Gamma\left(c_{j}\right)}-\frac{1}{2} \log (32 \Omega)\right\}+O\left(\sigma^{2}\right)=0,
\end{aligned}
$$

the values of $n$ concerned being such as make the initial term small. The formulas which are valid in this case, i.e., when $\Delta$ and $\Omega$ are nearly equal, are thus

$$
\begin{aligned}
& S_{n}(\Omega)=\Omega+(32 \Omega)^{1 / 2}\left\{\frac{\left(n-\frac{1}{4}\right) \pi-(8 \Omega)^{1 / 2}}{\log (32 \Omega)-2 \Gamma^{\prime}\left(\frac{3}{4}\right) / \Gamma\left(\frac{3}{4}\right)}\right\}+O(1), \\
& C_{n}(\Omega)=\Omega+(32 \Omega)^{1 / 2}\left\{\frac{\left(n+\frac{1}{4}\right) \pi-(8 \Omega)^{1 / 2}}{\log (32 \Omega)-2 \Gamma^{\prime}\left(\frac{1}{4}\right) / \Gamma\left(\frac{1}{4}\right)}\right\}+O(1) .
\end{aligned}
$$

In particular, the values of $\Omega$ for which $\sigma=0$ is a root, i.e., for which there is a characteristic value equal to $\Omega$, are found to be as follows:

$$
\begin{aligned}
& \text { If } S_{n}(\Omega)=\Omega, \text { then }(2 \Omega)^{1 / 2}=\left(n-\frac{1}{4}\right) \frac{\pi}{2}+O\left(\frac{\log n}{n}\right), \\
& \text { If } C_{n}(\Omega)=\Omega, \text { then }(2 \Omega)^{1 / 2}=\left(n+\frac{1}{4}\right) \frac{\pi}{2}+O\left(\frac{\log n}{n}\right)^{*} .
\end{aligned}
$$

* These values were considered by Goldstein, S., in A note on certain approximate solutions of linear differential equations, etc., Proceedings of the London Mathematical Society, (2), vol. 28(1928), p. 87, where the results are stated in the following form:

$$
\text { If } S_{n}(\Omega)=\Omega \text {, then }\left\{\begin{array}{l}
2^{1 / 2} \cos (8 \Omega)^{1 / 2} \sim(-1)^{n}, \\
2^{1 / 2} \sin (8 \Omega)^{1 / 2} \sim(-1)^{n+1}
\end{array}\right.
$$


Finally near the lower end of its permitted range $\sigma$ is large but negative, and the second of formulas (4.14) gives

$$
\gamma_{j}=-\sigma+\sigma \log |\sigma|-\left(2 c_{j}-1\right) \frac{\pi}{4}+O\left(\frac{1}{|\sigma|}\right) .
$$

The characteristic equations (4.13) accordingly become respectively

$$
(2 \Omega)^{1 / 2}+\frac{\sigma}{2} \log \frac{32 \Omega}{\sigma^{2}}+\sigma+\frac{\pi}{4}+O\left(\Omega^{-1 / 2} \log \Omega\right)+O\left(\frac{\Omega^{1 / 2}}{\Omega-\Delta}\right)=\frac{n \pi}{2},
$$

for the characteristic value $S_{n}(\Omega)$;

$$
(2 \Omega)^{1 / 2}+\frac{\sigma}{2} \log \frac{32 \Omega}{\sigma^{2}}+\sigma-\frac{\pi}{4}+O\left(\Omega^{-1 / 2} \log \Omega\right)+O\left(\frac{\Omega^{1 / 2}}{\Omega-\Delta}\right)=\frac{n \pi}{2},
$$

for the characteristic value $C_{n}(\Omega)$. These are, therefore, the forms which are valid when $\Omega$ is near the upper end of its permitted range of values, or, in other words, when $\Delta$ is near the lower end of its possible range.

4.6. The characteristic exponent. The formulas (4.6d) and (4.10b) yield for the evaluation of $\Theta$ in $(25 b)$

$$
\Theta=4 e^{-\sigma \pi}\left[\frac{\pi}{\Gamma_{1} \Gamma_{2}}\right]_{1} \cos \varepsilon_{1} \cos \varepsilon_{2}-1,
$$

where $\varepsilon_{1}$ and $\varepsilon_{2}$ are as defined in (4.11). The third of the formulas (4.14) may be made to give further

$$
\frac{\pi}{\Gamma_{1} \Gamma_{2}}=\left\{\frac{\cosh 2 \sigma \pi}{2}\right\}^{1 / 2}
$$

whence

$$
\Theta=2\left\{1+e^{-4 \sigma \pi}\right\}^{1 / 2}[1]_{1} \cos \varepsilon_{2} \cos \varepsilon_{1}-1,
$$

and the characteristic exponent is obtainable from the appropriate formula (25a).

The $(\Omega, \Delta)$ sub-regions of the domain IV of Figure 1 which comprise parameter values for which the differential equation has stable solutions are those for which the value of $\Theta$ is less than unity. It is evident from the formula (4.16) that these sub-regions become more and more attenuated as $\sigma$ decreases, i.e., as the right-hand boundary of the configuration IV is approached. 


\section{Chapter 5}

\section{The CONFiguration $\mathrm{V}$}

5.1. Preliminaries. Abutting the configuration of the preceding chapter is that denoted by $\mathrm{V}$ in Figure 1, in which $\Omega$ is taken to be large and

$$
0 \leqq \Delta \leqq \Omega-M_{2} \Omega^{1 / 2} \text {. }
$$

In this case the substitutions

$$
\rho=\frac{\Omega-\Delta}{\Omega^{1 / 2}}, \quad \sigma^{2}=1-\frac{\Delta}{\Omega}, \quad s=\frac{z}{\sigma}
$$

reduce the differential equation (1) to the form (3) with

$$
\begin{aligned}
\chi_{0}^{2}(s, \sigma) & \equiv \frac{2 \sin ^{2} \sigma s}{\sigma^{2}}-1, \\
\chi_{1} & \equiv 0 .
\end{aligned}
$$

The parameter $\rho$ is bounded below by the constant $M_{2}$ while $\sigma^{2}$ is confined to the fixed closed range $0 \leqq \sigma^{2} \leqq 1$, its smallest possible value being in fact $M_{2} \Omega^{-1 / 2}$.

With the strip (13) chosen as $R_{z}$, the region $R_{\varepsilon}$ is

$$
R_{8}: \quad 0 \leqq s^{\prime} \leqq \frac{\pi}{2 \sigma},
$$

and within this $\chi_{0}^{2}$ admits just one zero which is simple and is located on the axis of reals at the point

$$
s_{0}^{\prime}=\frac{1}{\sigma} \sin ^{-1} \frac{\sigma}{2^{1 / 2}} .
$$

The position of $s_{0}^{\prime}$ varies with $\sigma$ but is restricted to the fixed interval $\left(2^{-1 / 2}\right.$, $\pi / 4)$.

If $R_{s}$ is thought of as cut along the axis of reals from $s=0$ to $s=s_{0}^{\prime}$, the values of $\Phi$ on its boundaries are the following:

For $s^{\prime \prime}=0+, 0 \leqq s^{\prime} \leqq s_{0}^{\prime}$,

$$
\Phi=e^{\pi i} \int_{\sigma^{\prime}}^{s_{0^{\prime}}} i\left\{1-\frac{2 \sin ^{2} \sigma s^{\prime}}{\sigma^{2}}\right\}^{1 / 2} d s^{\prime} .
$$

For $s^{\prime}=0, s^{\prime \prime} \geqq 0$,

$$
\Phi=\Phi(0)+e^{\pi i / 2} \int_{0}^{\iota^{\prime}} i\left\{\frac{2 \sinh ^{2} \sigma s^{\prime \prime}}{\sigma^{2}}+1\right\}^{1 / 2} d s^{\prime} .
$$


For $s^{\prime}=\pi /(2 \sigma), s^{\prime \prime} \geqq 0$,

$$
\Phi=\Phi\left(\frac{\pi}{2 \sigma}\right)+e^{\pi i / 2} \int_{0}^{\Theta^{\prime \prime}}\left\{\frac{2 \cosh ^{2} \sigma s^{\prime \prime}}{\sigma^{2}}-1\right\}^{1 / 2} d s^{\prime \prime} .
$$

The maps of $R_{\varepsilon}$ upon $R_{\Phi}$, and hence of $R_{z}$ upon $R_{\xi}$, are thus revealed, the latter being as indicated in Figure 7:
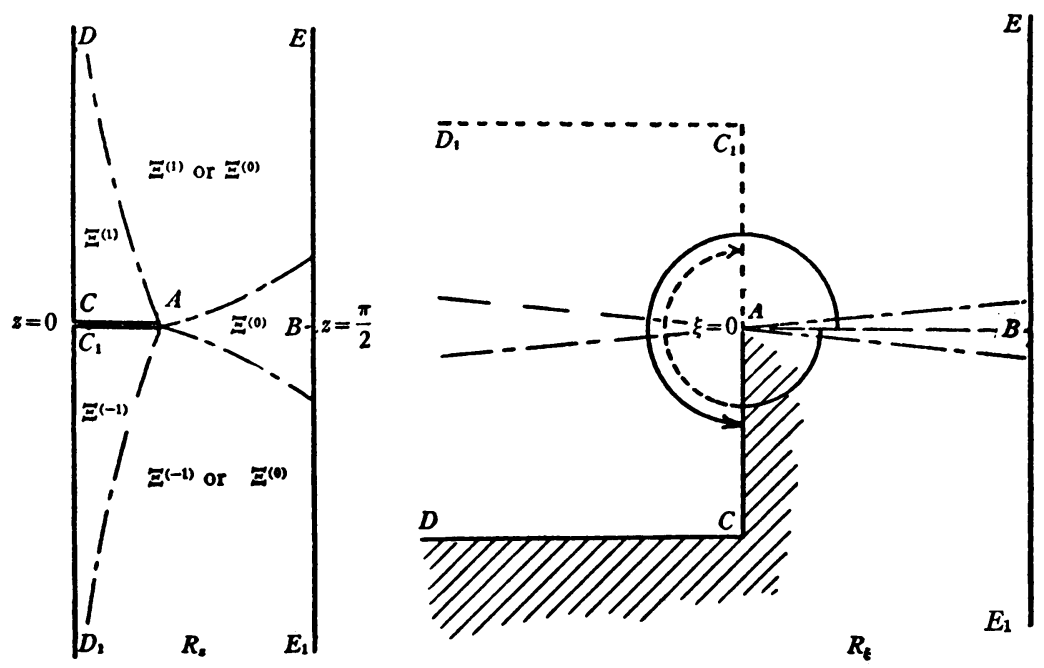

Fig. 7

5.2. The hypotheses. The discussion by which the uniform fulfillment of the requirements of $\$ 1.2$ by the present differential equation may be established, will be omitted as to detail inasmuch as it proceeds almost entirely like that of \$3.3. In virtue of the values (5.3) the functions (6) are in this case explicitly

$$
\begin{aligned}
\omega(\phi) & =\frac{1}{4}\left\{-\frac{5}{9}\left(\frac{\phi}{\Phi}\right)^{2}-\sigma^{2}+\frac{6\left(1-\sigma^{2}\right)}{\phi^{2}}+\frac{5\left(2-\sigma^{2}\right)}{\phi^{4}}\right\}, \\
\Psi & =\Phi^{1 / 6} / \phi^{1 / 2}, \quad \omega_{1} \equiv 0 .
\end{aligned}
$$

When $\left|s-s_{0}^{\prime}\right|$ is great the formulas

$$
\phi \sim 2^{1 / 2} \frac{\sin \sigma s}{\sigma}, \quad \Phi \sim \frac{2^{3 / 2}}{\sigma^{2}} \sin ^{2} \frac{\sigma s}{2}
$$

may be used, while for intermediate values the first of the formulas (5.3) may be written 


$$
\phi^{2}=2\left(1-\sigma^{2}\right) \frac{\sin ^{2} \sigma\left(s-s_{0}^{\prime}\right)}{\sigma^{2}}+\left(2-\sigma^{2}\right) \frac{\sin 2 \sigma\left(s-s_{0}^{\prime}\right)}{\sigma} .
$$

For small values of $\left|s-s_{0}^{\prime}\right|$ it may be shown that

$$
\Phi=\frac{\phi^{3}}{3\left(2-\sigma^{2}\right)^{1 / 2}}\left\{1-\frac{3\left(1-\sigma^{2}\right)}{5\left(2-\sigma^{2}\right)} \phi^{2}+\phi^{4} O(1)\right\},
$$

and with these formulas at hand the arguments of $\$ 3.3$ may be paralleled.

5.3. The solutions relative to $z=0$. The point $z=0$ may, as is seen from Figure 7, be regarded as lying in the sub-region $\Xi^{(1)}$. Moreover, the zero of $\chi_{0}{ }^{2}$ being simple the formulas of $\$ 1.4$ are applicable, with $h=1$ as the appropriate value. The formulas (11b) and (11a) thus become, in the manner now familiar, the following:

When $|\xi| \leqq N$,

$$
u_{o}(z)=\left\{\frac{\pi \sigma^{2} i}{6 \rho^{2} \phi_{1} \phi}\right\}^{1 / 2} \xi^{1 / 6} e^{i \xi_{1}}\left[\xi^{1 / 3} J_{-1 / 3}(\xi)+\xi^{1 / 3} J_{1 / 3}(\xi)\right],
$$

$$
u_{\odot}(z)=\left\{\frac{\pi \phi_{1}}{6 i \phi}\right\}^{1 / 2} \xi^{1 / 6} e^{i \xi_{1}}\left[\xi^{1 / 3} J_{-1 / 3}(\xi)+\xi^{1 / 3} J_{1 / 3}(\xi)\right] ;
$$

and when $z$ lies in $\Xi^{(l)}$, and $|\xi| \geqq N$,

\begin{tabular}{|c|c|c|c|}
\hline$l$ & -1 & 0 & 1 \\
\hline$K_{0,1}^{(l)}$ & $e^{i \xi_{1}[1]}$ & $e^{i \xi_{1}[1]}$ & $-i e^{-i \xi_{1}[1]}$ \\
\hline$K_{0,2}^{(l)}$ & $-e^{-i \xi_{1}[1]}$ & $i e^{i \xi_{1}[1]}$ & $i e^{i \xi_{1}[1]}$ \\
\hline$K_{0,1}^{(l)}$ & $-i e^{i \xi_{1}[1]}$ & $-i e^{i \xi_{1}[1]}$ & $e^{-i \xi_{1}[1]}$ \\
\hline$K_{0,2}^{(l)}$ & $-i e^{-i \xi_{1}[1]}$ & $e^{i \xi_{1}[1]}$ & $e^{i \xi_{1}[1]}$ \\
\hline
\end{tabular}

$$
\begin{aligned}
& u_{o}(z)=\frac{1}{2}\left\{\frac{\sigma^{2}}{\rho^{2} \phi_{1} \phi}\right\}^{1 / 2}\left\{K_{0,1}^{(l)} e^{i \xi}+K_{0,2}^{(l)} e^{-i \xi}\right\}, \\
& u_{e}(z)=\frac{1}{2}\left\{\frac{\phi_{1}}{\phi}\right\}^{1 / 2}\left\{K_{e, 1}^{(l)} e^{i \xi}+K_{e, 2}^{(l)} e^{-i \xi}\right\},
\end{aligned}
$$

with coefficients 
The symbols involved would have the evaluations

$$
\begin{aligned}
\frac{\rho \phi}{\sigma} & =\{\Delta-\Omega \cos 2 z\}^{1 / 2}, \quad \frac{\rho \phi_{1}}{\sigma}=e^{\pi i / 2}\{\Omega-\Delta\}^{1 / 2}, \\
\xi & =\int_{x_{0}}^{z}\{\Delta-\Omega \cos 2 z\}^{1 / 2} d z, \quad x_{0}=\frac{1}{2} \cos ^{-1} \Delta / \Omega, \\
\xi_{1} & =e^{3 \pi i / 2} \int_{0}^{x_{0}}\{\Omega \cos 2 x-\Delta\}^{1 / 2} d x .
\end{aligned}
$$

When $z$ is real and less than $x_{0}$ the relation $\xi=|\xi| e^{8 \pi i / 2}$ is valid and hence

$$
J_{-1 / 3}(\xi)+J_{1 / 3}(\xi)=\frac{3^{1 / 2}}{\pi i} K_{1 / 8}(|\xi|) .
$$

The formulas given in (5.5) thus reduce when the variable is real to the following:

When $0 \leqq x<x_{0}$, and $|\xi| \geqq N$,

$$
\begin{aligned}
& u_{0}(x)=\frac{[1]}{\{(\Omega-\Delta)(\Omega \cos 2 x-\Delta)\}^{1 / 4}} \sinh \left[\int_{0}^{x}\{\Omega \cos 2 x-\Delta\}^{1 / 2} d x\right] \\
& u_{0}(x)=\left\{\frac{\Omega-\Delta}{\Omega \cos 2 x-\Delta}\right\}^{1 / 4}[1] \cosh \left[\int_{0}^{x}\{\Omega \cos 2 x-\Delta\}^{1 / 2} d x\right] .
\end{aligned}
$$

When $x \leqq x_{0}$, and $|\xi| \leqq N$,

$$
u_{0}(x)=\frac{|\xi|^{1 / 6} e^{\left|\xi_{1}\right|}}{\left\{4 \pi^{2}(\Omega-\Delta)(\Omega \cos 2 x-\Delta)\right\}^{1 / 4}}\left[|\xi|^{1 / 3} K_{1 / 8}(|\xi|)\right],
$$

$$
\text { with }|\xi|=\int_{x}^{x_{0}}\{\Omega \cos 2 x-\Delta\}^{1 / 2} d x \text {. }
$$

When $x_{0} \leqq x$, and $|\xi| \leqq N$,

(5.6c) $\quad u_{0}(x)=\frac{(\pi / 6)^{1 / 2} \xi^{1 / 6} e^{\left|\xi_{1}\right|}}{\{(\Omega-\Delta)(\Delta-\Omega \cos 2 x)\}^{1 / 4}}\left[\xi^{1 / 3} J_{-1 / 3}(\xi)+\xi^{1 / 3} J_{1 / 3}(\xi)\right]$.

When $x_{0}<x \leqq \pi / 2$, and $|\xi| \geqq N$,

$$
\begin{aligned}
u_{0}(x)=\frac{[1] e^{|\xi|}}{\{(\Omega-\Delta)(\Delta-\Omega \cos 2 x)\}^{1 / 4}} & \cdot \sin \left[\frac{\pi}{4}+\int_{x_{0}}^{\cdot x}\{\Delta-\Omega \cos 2 x\}^{1 / 2} d x\right] .
\end{aligned}
$$


In the cases (b), (c) and (d) the representation of $u_{0}(x)$ may be formally obtained from that of $u_{0}(x)$ by replacing the factor $(\Omega-\Delta)^{-1 / 4}$ by $(\Omega-\Delta)^{1 / 4}$.

5.4. The solutions relative to $z=\pi / 2$. The formulas (11) with the subscripts $a$ replaced by 2 , where the latter denote values corresponding to $z=\pi / 2$, may be made to yield also the solutions $u_{\alpha}(z)$ and $u_{\beta}(z)$. Since the point $z=\pi / 2$ lies in the sub-region $\Xi^{(0)}$ the value $h=0$ is appropriate and the formulas obtained are the following:

When $|\xi| \leqq N$,

$$
\begin{aligned}
u_{\alpha}(z)=\left(\frac{2 \pi \sigma^{2}}{3 \rho^{2} \phi_{2} \phi}\right)^{1 / 2} \xi^{1 / 6}\left\{\operatorname { c o s } \left(\xi_{2}\right.\right. & \left.-\frac{\pi}{12}\right)\left[\xi^{1 / 3} J_{1 / 3}(\xi)\right] \\
& \left.-\sin \left(\xi_{2}+\frac{\pi}{12}\right)\left[\xi^{1 / 3} J_{-1 / 3}(\xi)\right]\right\},
\end{aligned}
$$

(5.7a)

$$
\begin{aligned}
u_{\beta}(z)=\left(\frac{2 \pi \phi_{2}}{3 \phi}\right)^{1 / 2} \xi^{1 / 6}\left\{\operatorname { s i n } \left(\xi_{2}-\right.\right. & \left.\frac{\pi}{12}\right)\left[\xi^{1 / 3} J_{1 / 3}(\xi)\right] \\
& \left.+\cos \left(\xi_{2}+\frac{\pi}{12}\right)\left[\xi^{1 / 3} J_{-1 / 3}(\xi)\right]\right\} .
\end{aligned}
$$

When $z$ is in $\Xi^{(l)}$, and $|\xi| \geqq N$,

$$
\begin{aligned}
& u_{\alpha}(z)=\frac{1}{2}\left(\frac{\sigma^{2}}{\rho^{2} \phi_{2} \phi}\right)^{1 / 2}\left\{K_{\alpha, 1}^{(l)} e^{i \xi}+K_{\alpha, 2}^{(l)} e^{-i \xi}\right\}, \\
& u_{\beta}(z)=\frac{1}{2}\left(\frac{\phi_{2}}{\phi}\right)^{1 / 2}\left\{K_{\beta, 1}^{(l)} e^{i \xi}+K_{\beta, 2}^{(l)} e^{-i \xi}\right\}
\end{aligned}
$$

with coefficients

\begin{tabular}{c|c|c|c|}
\hline$l$ & -1 & 0 & 1 \\
\hline$K_{\alpha, 1}^{(l)}$ & $-i e^{-i \xi_{2}[1]}$ & $-i e^{i \xi_{2}[1]}$ & $2 e^{-3 \pi i / 4}\left[\cos \left(\xi_{2}-\frac{\pi}{4}\right)\right]$ \\
\hline$K_{\alpha, 2}^{(l)}$ & $2 e^{3 \pi i / 4}\left[\cos \left(\xi_{2}-\frac{\pi}{4}\right)\right]$ & $i^{i \xi_{2}[1]}$ & $i e^{i \xi_{2}[1]}$ \\
\hline$K_{\beta, 1}^{(l)}$ & $2 e^{3 \pi i / 4}\left[\sin \left(\xi_{2}-\frac{\pi}{4}\right)\right]$ & $e^{i \xi_{2}[1]}$ & $e^{i \xi_{2}[1]}$ \\
\hline$K_{\beta, 2}^{(l)}$ & $e^{-i \xi_{2}[1]}$ & $e^{-i \xi_{2}[1]}$ & $2 e^{-8 \pi i / 4}\left[\sin \left(\xi_{2}-\frac{\pi}{4}\right)\right]$ \\
\hline
\end{tabular}


In terms of the original variables

$$
\begin{aligned}
\frac{\rho \phi_{2}}{\sigma} & =\{\Omega+\Delta\}^{1 / 2}, \\
\xi_{2} & =\int_{x_{0}}^{\pi / 2}\{\Delta-\Omega \cos 2 x\}^{1 / 2} d x .
\end{aligned}
$$

The forms obtained for real values of the variable are the following: When $0 \leqq x<x_{0}$, and $|\xi| \geqq N$,

$$
\begin{array}{r}
u_{\alpha}(x)=\frac{-\left[\cos \left(\frac{\pi}{4}-\xi_{2}\right)\right]}{\{(\Omega+\Delta)(\Omega \cos 2 x-\Delta)\}^{1 / 4}} \exp \left(\int_{x}^{x_{0}}\{\Omega \cos 2 x-\Delta\}^{1 / 2} d x\right), \\
u_{\beta}(x)=\left\{\frac{\Omega+\Delta}{\Omega \cos 2 x-\Delta}\right\}^{1 / 4}\left[\sin \left(\frac{\pi}{4}-\xi_{2}\right)\right] \\
\cdot \exp \left(\int_{x}^{x_{0}}\{\Omega \cos 2 x-\Delta\}^{1 / 2} d x\right) .
\end{array}
$$

When $x \leqq x_{0}$, and $|\xi| \leqq N$,

$$
\begin{aligned}
u_{\alpha}(x)=\frac{-(2 \pi / 3)^{1 / 2}|\xi|^{1 / 6}}{\{(\Omega+\Delta)(\Omega \cos 2 x-\Delta)\}^{1 / 4}} & \left\{\cos \left(\xi_{2}-\frac{\pi}{12}\right)\left[|\xi|^{1 / 3} I_{1 / 3}(|\xi|)\right]\right. \\
& \left.+\sin \left(\xi_{2}+\frac{\pi}{12}\right)\left[|\xi|^{1 / 3} I_{-1 / 3}(|\xi|)\right]\right\},
\end{aligned}
$$

$$
\begin{array}{r}
u_{\beta}(x)=\left(\frac{2 \pi}{3}\right)^{1 / 2}|\xi|^{1 / 6}\left\{\frac{\Omega+\Delta}{\Omega \cos 2 x-\Delta}\right\}^{1 / 4}\left\{\sin \left(\xi_{2}-\frac{\pi}{12}\right)\left[|\xi|^{1 / 3} I_{1 / 3}(|\xi|)\right]\right. \\
\left.-\cos \left(\xi_{2}+\frac{\pi}{12}\right)\left[|\xi|^{1 / 3} I_{-1 / 3}(|\xi|)\right]\right\} .
\end{array}
$$

When $x_{0} \leqq x$, and $|\xi| \leqq N$,

$$
\begin{aligned}
& u_{\alpha}(x)=\frac{(2 \pi / 3)^{1 / 2} \xi^{1 / 6}}{\{(\Omega+\Delta)(\Delta-\Omega \cos 2 x)\}^{1 / 4}}\left\{\cos \left(\xi_{2}-\frac{\pi}{12}\right)\left[\xi^{1 / 3} J_{1 / 3}(\xi)\right]\right. \\
&\left.-\sin \left(\xi_{2}+\frac{\pi}{12}\right)\left[\xi^{1 / 3} J_{-1 / 3}(\xi)\right]\right\},
\end{aligned}
$$

$$
\begin{aligned}
u_{\beta}(x)=\left(\frac{2 \pi}{3}\right)^{1 / 2} \xi^{1 / 6}\left\{\frac{\Omega+\Delta}{\Delta-\Omega \cos 2 x}\right\}^{1 / 4}\{ & \sin \left(\xi_{2}-\frac{\pi}{12}\right)\left[\xi^{1 / 3} J_{1 / 8}(\xi)\right] \\
& \left.+\cos \left(\xi_{2}+\frac{\pi}{12}\right)\left[\xi^{1 / 3} J_{-1 / 8}(\xi)\right]\right\} .
\end{aligned}
$$


When $x_{0}<x \leqq \pi / 2$, and $|\xi| \geqq N$,

$$
\begin{aligned}
& u_{\alpha}(x)=\frac{[1]}{\{(\Omega+\Delta)(\Delta-\Omega \cos 2 x)\}^{1 / 4}} \sin \left[\int_{\pi / 2}^{x}\{\Delta-\Omega \cos 2 x\}^{1 / 2} d x\right] \\
& u_{\beta}(x)=\left\{\frac{\Omega+\Delta}{\Delta-\Omega \cos 2 x}\right\}^{1 / 4}[1] \cos \left[\int_{\pi / 2}^{x}\{\Delta-\Omega \cos 2 x\}^{1 / 2} d x\right] .
\end{aligned}
$$

5.5. The solutions of the associated equation. The positive axis of imaginaries in Figure 7 lies in the sub-region $\Xi^{(1)}$. The formulas (5.5) appropriate to this region are to be used, therefore, in obtaining the solutions of the equation (2) for real values of the variable by the substitutions (12). The formulas thus found are

$$
\begin{aligned}
& v_{0}(x)=\frac{[1]}{\{(\Omega-\Delta)(\Omega \cosh 2 x-\Delta)\}^{1 / 4}} \sin \left[\int_{0}^{x}\{\Omega \cosh 2 x-\Delta\}^{1 / 2} d x\right] \\
& v_{0}(x)=\left\{\frac{\Omega-\Delta}{\Omega \cosh 2 x-\Delta}\right\}^{1 / 4}[1] \cos \left[\int_{0}^{x}\{\Omega \cosh 2 x-\Delta\}^{1 / 2} d x\right] .
\end{aligned}
$$

5.6. The characteristic values and exponent. The forms (5.6d) show that the characteristic values for both even and odd Mathieu functions are in this case determined by equations

$$
\left[\frac{\pi}{4}+\int_{x_{0}}^{\pi / 2}\{\Delta-\Omega \cos 2 x\}^{1 / 2} d x\right]=\frac{n \pi}{2},
$$

the proper correlation of the indices of the roots with the integer $n$ being duly regarded.

If $k_{1}$ is defined in terms of $\Delta$ and $\Omega$ by the same formula as is the $\sigma$ of chapter 4 , i.e., by (3.15), the substitutions

$$
\cos x=h \sin \zeta, \quad h^{2}=1+\frac{4 k_{1}}{(2 \Omega)^{1 / 2}}
$$

reduce the equation $(5.10 \mathrm{a})$ to the form

$$
\frac{\pi}{4}+(2 \Omega)^{1 / 2} h^{2} G\left(1, h^{2}\right)+O\left(\frac{\Omega^{1 / 2}}{\Omega-\Delta}\right)=\frac{n \pi}{2},
$$

in which $G$ is the elliptic integral of (26). In the range of transition from the configuration of chapter 4 to that of the present chapter, $k_{1}$ is negative and $h^{2}$ accordingly little less than unity. The evaluation of $(5.10 \mathrm{~b})$ to the form

$$
\frac{\pi}{4}+(2 \Omega)^{1 / 2}+\frac{k_{1}}{2} \log \frac{32 \Omega}{k_{1}{ }^{2}}+k_{1}+O\left(\Omega^{-1 / 2} \log \Omega\right)+O\left(\frac{\Omega^{1 / 2}}{\Omega-\Delta}\right)=\frac{n \pi}{2}
$$


may, therefore, be obtained by the use of (26b), and a comparison of the result with the equations $(4.13 \mathrm{c})$ shows that the characteristic values which occur as the roots of equations representable by (5.10a) are respectively $S_{n}(\Omega)$ and $C_{n-1}(\Omega)$. In other words the characteristic equations are as follows:

$$
\int_{x_{0}}^{\pi / 2}\{\Delta-\Omega \cos 2 x\}^{1 / 2} d x+O\left(\frac{\Omega^{1 / 2}}{\Omega-\Delta}\right)=\left(n-\frac{1}{2}\right) \frac{\pi}{2},
$$
for the characteristic value $S_{n}(\Omega)$;

$$
\int_{x_{0}}^{\pi / 2}\{\Delta-\Omega \cos 2 x\}^{1 / 2} d x+O\left(\frac{\Omega^{1 / 2}}{\Omega-\Delta}\right)=\left(n+\frac{1}{2}\right) \frac{\pi}{2},
$$

for the characteristic value $C_{n}(\Omega)$.

In the consideration of the characteristic exponent the formulas (5.6d) and (5.8a) in conjunction with (25b) are found to lead to the evaluation

$$
\Theta=e^{2\left|\xi_{1}\right|}\left[\cos 2 \xi_{2}\right]-1,
$$

and with this the value of $\mu$ is given by the formula (25a). Since the righthand member of (5.12) can be exceeded by unity only when the cosine is very small, it is evident that the unstable solutions greatly predominate in the present configuration.

An evaluation of the several elliptic integrals involved may be made to show that the transition from the formula (4.16) to (5.12) is a continuous one.

\section{Chapter 6}

The CONFIGURATION VI

6.1. Remarks. The configuration designated by VI in Figure 1 is to be that in which $\Delta$ is negative and

$$
-\left\{\Omega-M_{2} \Omega^{1 / 2}\right\} \leqq \Delta \leqq 0 .
$$

It clearly differs from that of the preceding chapter only in the sign of $\Delta$. The distinction between the two configurations is indeed largely an artificial one, entered into primarily for the purpose of utilizing the discussion of $\$ 5.2$ without modification when parameter values admitted by (6.1) are concerned. For in this latter case the substitutions

$$
\rho=\frac{\Omega+\Delta}{\Omega^{1 / 2}} e^{3 \pi i / 2}, \quad \sigma^{2}=1+\frac{\Delta}{\Omega}, \quad s=\frac{1}{\sigma}\left(\frac{\pi}{2}-z\right)
$$

transform the differential equation (1) into the form (3) with precisely the coefficients (5.3), with $\sigma$ restricted precisely as in the earlier case. The de- 
ductions of $\$ 5.2$, therefore, serve again to show that the requirements of the general theory are uniformly fulfilled.

By their definitions the intermediate variables $s, \phi$, and $\Phi$, and the parameters $\sigma$ and $\rho$, differ from the corresponding quantities in chapter 5. The ultimate variables $z$ and $\xi$ are, however, found to have the same relation to each other, so that Figure 7 continues to remain valid in the present configuration. It is found as a consequence that the various formulas deduced in $\S 5.3, \S 5.4$, and $\$ 5.5$ apply also in the present instance, provided they are expressed entirely in terms of the original variables $z, \Delta$, and $\Omega$.

6.2. The characteristic values and exponent. With the prevailing forms of the solutions exactly those of chapter 5 the characteristic equations of course remain of the form (5.11). It is of interest, however, to obtain from these equations more explicit formulas which are valid near the lower end of the admitted range of values for $\Delta$. For such values $h^{2}$, which may be written

$$
h^{2}=\frac{\Omega-|\Delta|}{2 \Omega},
$$

is small of the order of $\Omega^{-1 / 2}$, and in the equation (5.10b) the evaluation given by (26a) is appropriate. The equation thus becomes

$$
(2 \Omega)^{1 / 2} h^{2}\left\{1+\frac{h^{2}}{8}+h^{4} O(1)\right\}=2 n-1 .
$$

It is evident that the integers $n$ concerned are those of a bounded set, the equation being expressible for such $n$ in the form

$$
\Omega+\Delta=(2 n-1)(2 \Omega)^{1 / 2}+O(1) .
$$

Inasmuch as the characteristic equations represented by $(5.10 \mathrm{~b})$ were found to be those for $S_{n}(\Omega)$ and $C_{n-1}(\Omega)$, it follows that for the algebraically smaller of the presently admitted values of $\Delta$ the characteristic values are described by formulas

$$
\begin{aligned}
& S_{n}(\Omega)=-\Omega+(2 n-1)(2 \Omega)^{1 / 2}+O(1), \\
& C_{n}(\Omega)=-\Omega+(2 n+1)(2 \Omega)^{1 / 2}+O(1) .
\end{aligned}
$$

The characteristic exponent is again given by (25a) and (5.12).

\section{Chapter 7}

\section{ThE CONFIGURATION VII}

7.1. The transformed differential equation. When $\Delta$ is large and negative and

$$
-M_{2}|\Delta|^{1 / 2} \leqq \Omega+\Delta \leqq M_{2} \Omega^{1 / 2},
$$


the configuration is that designated by VII, Figure 1 . In this case the substitutions

$$
\rho=(32 \Omega)^{1 / 2} e^{-x i / 2}, \quad \sigma=\frac{\Omega+\Delta}{(32 \Omega)^{1 / 2}}, \quad s=\frac{\pi}{2}-z
$$

bring the differential equation (1) into the form (3) with

$$
\begin{aligned}
\chi_{0}(s, \sigma) & \equiv \frac{1}{4} \sin s, \\
\chi_{1} & \equiv i \sigma .
\end{aligned}
$$

The transformed equation thus differs from that obtained in chapter 4 only to the extent that $\sigma$ is replaced by $i \sigma$. The formulas for $\phi$ and $\Phi$ given in (4.4) are adaptable to the present case by the substitution of $-\sigma /|\rho|$ in place of $\sigma / \rho$, a change which is easily seen to affect in no way the validity of the arguments of $\$ 4.1$. That the differential equation in the present instance uniformly satisfies the hypotheses of $\$ 1.2$ may, therefore, be accepted without further consideration.

The regions $R_{\mathrm{f}}$ and $R_{\Phi}$ which correspond to the strip (13) are, both as to outline and relative orientation, precisely like the $z$ and $\xi$ regions shown in Figure 5. Since under the relations (7.2) the region $R_{\mathrm{s}}$ is a reflection of $R_{\mathrm{s}}$ in the point $s=\pi / 4$, whereas $R_{\xi}$ is obtainable from $R_{\Phi}$ by a rotation besides the change of scale, the figure which relates the ultimate regions $R_{\mathrm{z}}$ and $R_{\xi}$ for the chapter at hand is as indicated in Figure 8. The division of these regions into the sub-regions $\Xi^{(l)}$ is also as shown.

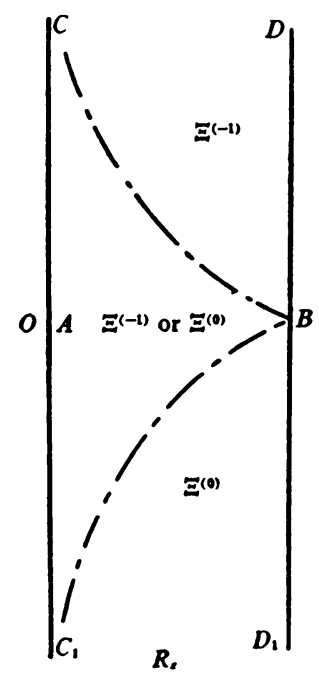

$\boldsymbol{R}$

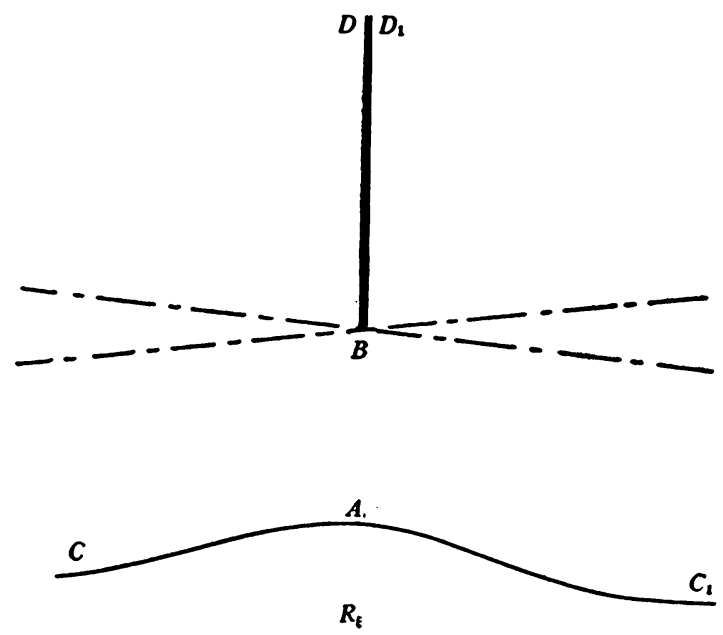

Frg. 8 
7.2. The solutions. The origin $z=0$ corresponds to $s_{1}=\pi / 2$ and lies in the sub-region $\Xi^{(0)}$. The principal solutions relative to this point may accordingly be deduced by the substitution of the values (4.9) (with $\sigma$ replaced by $i \sigma$ ) into the formulas (8b), the subscript $a$ being taken as 1 . To this extent the process coincides with that by which the forms (4.10) were deduced. In the present instance, however, certain terms may be dropped from the resulting formulas for, as may be seen from Figure 8 , the quantity $i \xi_{1}$ is real and positive and $e^{-i \xi_{1}}$ therefore asymptotically negligible in comparison with $e^{i \xi_{1}}$. It is found thus that the following formulas hold:

When $z$ is (anywhere) in $R_{z}$, and $|\xi| \geqq N$,

$$
u_{o}(z)=\frac{-1}{2 i \rho \phi_{1}{ }^{1 / 2} \phi^{1 / 2}}\left\{\left(\frac{\xi}{\xi_{1}}\right)^{-\sigma} e^{i\left(\xi-\xi_{1}\right)}[1]_{1}-\left(\frac{\xi}{\xi_{1}}\right)^{\sigma} e^{-i\left(\xi-\xi_{1}\right)}[1]_{1}\right\},
$$

$$
u_{e}(z)=\frac{\phi_{1}^{1 / 2}}{2 \phi^{1 / 2}}\left\{\left(\frac{\xi}{\xi_{1}}\right)^{-\sigma} e^{i\left(\xi-\xi_{1}\right)}[1]_{1}+\left(\frac{\xi}{\xi_{1}}\right)^{\sigma} e^{-i\left(\xi-\xi_{1}\right)}[1]_{1}\right\} ;
$$

when $|\xi| \leqq N$,

$$
\begin{aligned}
u_{o}(z)=\left(\frac{\pi}{\phi_{1} \phi}\right)^{1 / 2} \frac{\left(2 i \xi_{1}\right)^{-\sigma} e^{i \xi_{1}}(i \xi)^{1 / 4}}{2 i \rho}\left\{\frac{2 e^{\pi_{i} / 4}}{\Gamma\left(\frac{1}{4}-\sigma\right)}\right. & {\left[\mathcal{X}_{1}(\xi, i \sigma)\right]_{1} } \\
& \left.-\frac{1}{\Gamma\left(\frac{3}{4}-\sigma\right)}\left[\mathcal{X}_{2}(\xi, i \sigma)\right]\right\},
\end{aligned}
$$

$$
\begin{aligned}
u_{e}(z)=-\left(\frac{\pi \phi_{1}}{\phi}\right)^{1 / 2} \frac{\left(2 i \xi_{1}\right)^{-\sigma} e^{i \xi_{1}(i \xi)^{1 / 4}}}{2}\left\{\frac{2 e^{\pi i / 4}}{\Gamma\left(\frac{1}{4}-\sigma\right)}\left[\mathcal{X}_{1}(\xi, i \sigma)\right]_{1}\right. \\
\left.-\frac{1}{\Gamma\left(\frac{3}{4}-\sigma\right)}\left[\mathcal{X}_{2}(\xi, i \sigma)\right]_{1}\right\} .
\end{aligned}
$$

In these as in subsequent formulas any term is to be omitted if $\sigma$ is such that the gamma function involved is infinite.

The point $z=\pi / 2$ corresponds to $s_{2}=0$ and the principal solutions relative to this point are therefore to be obtained precisely as were the solutions of §4.2. The formulas found are as follows:

When $|\xi| \leqq N$,

$$
u_{\alpha}(z)=-\left(\frac{2}{i_{\rho}}\right)^{1 / 2} \Psi\left[(i \xi)^{-1 / 4} M_{\sigma, 1 / 4}(2 i \xi)\right],
$$

$$
u_{\beta}(z)=\left(\frac{1}{2}\right)^{1 / 2} \Psi\left[(i \xi)^{-1 / 4} M_{\sigma,-1 / 4}(2 i \xi)\right]
$$


when $|\xi| \geqq N$, and $z$ is in $\Xi^{(l),}$

$$
\begin{aligned}
u_{\alpha}(z)=-\left(\frac{\pi}{2 \phi}\right)^{1 / 2}(i \rho)^{-1 / 4}\left\{\left[\frac{1}{\Gamma\left(\frac{3}{4}-\sigma\right)}\right]_{1}\right. & (2 i \xi)^{-\sigma} e^{i \xi} \\
& \left.+\left[\frac{h_{\alpha}^{(1)}}{\Gamma\left(\frac{3}{4}+\sigma\right)}\right]_{1}(2 i \xi)^{\sigma} e^{-i \xi}\right\},
\end{aligned}
$$

$$
\begin{aligned}
u_{\beta}(z)=\left(\frac{\pi}{2 \phi}\right)^{1 / 2}(i \rho)^{-1 / 4}\left\{\left[\frac{1}{\Gamma\left(\frac{1}{4}-\sigma\right)}\right]_{1}\right. & (2 i \xi)^{-\sigma} e^{i \xi} \\
& \left.+\left[\frac{h_{\beta}^{(l)}}{\Gamma\left(\frac{1}{4}+\sigma\right)}\right]_{1}(2 i \xi)^{\sigma} e^{-i \xi}\right\},
\end{aligned}
$$

with coefficients given by the table

\begin{tabular}{c|c|c|}
\hline & -1 & 0 \\
\hline$h_{\alpha}^{(l)}$ & $e^{(\sigma-8 / 4) \pi i}$ & $e^{-(\sigma-8 / 4) \pi i}$ \\
\hline$h_{\beta}^{(l)}$ & $e^{(\sigma-1 / 4) \pi i}$ & $\sigma^{-(\sigma-1 / 4) \pi i}$ \\
\hline
\end{tabular}

In terms of the original variables

$$
\begin{aligned}
& \phi=\frac{\cos z}{4}-\frac{\Omega+\Delta}{32 \Omega} \tan \left(\frac{\pi}{4}-\frac{z}{2}\right) \\
& i \xi=(2 \Omega)^{1 / 2}(1-\sin z)+\frac{\Omega+\Delta}{(32 \Omega)^{1 / 2}} \log \frac{1+\sin z}{2},
\end{aligned}
$$

which permits the abbreviated relations

$$
\begin{aligned}
\phi & =\left[\frac{\cos z}{4}\right], & \phi_{1} & =\left[\frac{1}{4}\right], \\
i \xi & =\frac{|\rho|}{4}[1-\sin z], & i \xi_{1} & =\frac{|\rho|}{4}[1], \\
e^{i \xi} & =\left(\frac{1+\sin z}{2}\right)^{\sigma} e^{(|\rho| / 4)(1-\sin z)} & e^{i \xi_{1}} & =\left(\frac{1}{2}\right)^{\sigma} e^{|\rho| / 4} .
\end{aligned}
$$

The specialization of the various formulas to the case in which the variable is real may be made as usual, it being noted that then $i \xi=|\xi|$. The representations which result are as follows: 
When $|\xi| \geqq N$

(7.6a)

$$
u_{0}(x)=\left(\frac{\sec x}{2 \Omega}\right)^{1 / 2}[1]_{1} \sinh \left[(2 \Omega)^{1 / 2} \sin x+\sigma \log \frac{1+\sin x}{1-\sin x}\right]_{1},
$$

when $|\xi| \leqq N$,

$$
u_{e}(x)=(\sec x)^{1 / 2}[1]_{1} \cosh \left[(2 \Omega)^{1 / 2} \sin x+\sigma \log \frac{1+\sin x}{1-\sin x}\right]_{1} ;
$$

$$
u_{0}(x)=\frac{-(2 \pi)^{1 / 2} e^{(2 \Omega))^{1 / 2}}}{(32 \Omega)^{\sigma / 2+3 / 8}(1+\sin x)^{1 / 4}}\left\{\frac{2}{\Gamma\left(\frac{1}{4}-\sigma\right)}\left[|\xi|^{-1 / 4} M_{\alpha, 1 / 4}(2|\xi|)\right]_{1}\right.
$$

$$
\begin{aligned}
- & \left.\frac{1}{\Gamma\left(\frac{3}{4}-\sigma\right)}\left[|\xi|^{-1 / 4} M_{\alpha,-1 / 4}(2|\xi|)\right]_{1}\right\}, \\
u_{e}(x)=\frac{-(2 \pi)^{1 / 2} e^{(2 \Omega)^{1 / 2}}}{(32 \Omega)^{\sigma / 2-1 / 8}(1+\sin x)^{1 / 4}} & \left\{\frac{2}{\Gamma\left(\frac{1}{4}-\sigma\right)}\left[|\xi|^{-1 / 4} M_{\alpha, 1 / 4}(2|\xi|)\right]_{1}\right. \\
- & \left.\frac{1}{\Gamma\left(\frac{3}{4}-\sigma\right)}\left[|\xi|^{-1 / 4} M_{\alpha,-1 / 4}(2|\xi|)\right]_{1}\right\},
\end{aligned}
$$

the symbols $M$ representing the confluent hypergeometric functions which occur in the formulas (4.5).

When $|\xi| \geqq N$,

$$
\begin{aligned}
& u_{\alpha}(x)=\frac{-(2 \pi \sec x)^{1 / 2}}{(32 \Omega)^{\sigma / 2+3 / 8}}\left(\frac{1+\sin x}{1-\sin x}\right)^{\sigma}\left[\frac{1}{\Gamma\left(\frac{3}{4}-\sigma\right)}\right]_{1}^{e^{(2 \Omega))^{1 / 2}(1-\sin x)}} \\
& u_{\beta}(x)=\frac{(2 \pi \sec x)^{1 / 2}}{(32 \Omega)^{\sigma / 2+1 / 8}}\left(\frac{1+\sin x}{1-\sin x}\right)^{\sigma}\left[\frac{1}{\Gamma\left(\frac{1}{4}-\sigma\right)}\right]_{1}^{e^{(2 \Omega))^{1 / 2}(1-\sin x)}}
\end{aligned}
$$

When $|\xi| \leqq N$,

$$
u_{\alpha}(x)=\frac{-1}{(8 \Omega)^{1 / 2}(1+\sin x)^{1 / 4}}\left[|\xi|^{-1 / 4} M_{\alpha, 1 / 4}(2|\xi|)\right]
$$

$$
u_{\beta}(x)=\frac{1}{(1+\sin x)^{1 / 4}}\left[|\xi|^{-1 / 4} M_{\alpha,-1 / 4}(2|\xi|)\right] .
$$

The solutions of the associated Mathieu equation, as obtained from the forms $(7.4 \mathrm{a})$ by the method of $\$ 1.5$, are for real values of the variable represented thus:

$$
\begin{aligned}
& v_{o}(x)=\frac{[1]_{1}}{(2 \Omega \cosh x)^{1 / 2}} \sin \left[(2 \Omega)^{1 / 2} \sinh x-2 \sigma \tan ^{-1}(\sinh x)\right]_{1}, \\
& v_{e}(x)=\frac{[1]_{1}}{(\cosh x)^{1 / 2}} \cos \left[(2 \Omega)^{1 / 2} \sinh x-2 \sigma \tan ^{-1}(\sinh x)\right]_{1} .
\end{aligned}
$$


7.3. The characteristic values and exponent. The characteristic equations (17) and (18) may obviously if desired be rewritten in the forms $u_{\alpha}(0)=0, u_{\beta}(0)=0$ and $u_{\beta}^{\prime}(0)=0, u_{\alpha}^{\prime}(0)=0$. It accordingly follows from the formulas (7.7a) that any characteristic value must be a root of the one or the other of the equations

$$
\left[\frac{1}{\Gamma\left(\frac{3}{4}-\sigma\right)}\right]_{1}=0, \quad\left[\frac{1}{\Gamma\left(\frac{1}{4}-\sigma\right)}\right]_{1}=0 .
$$

If $\sigma$ is not positive the relations (7.9) are manifestly impossible. Hence no characteristic values exist when $\Delta \leqq-\Omega$, a fact which may be simply concluded from a direct perusal of the differential equation. When $\sigma$ is positive and of suitable magnitude, on the other hand, a relation (7.9) may be satisfied in virtue of the gamma function becoming infinite. The appropriate values are clearly those for which

$$
\left[\sigma+\frac{1}{4}\right]_{1}=\frac{n}{2}
$$

whence the characteristic equations are found to be of the form

$$
\Delta=-\Omega+(2 n-1)(2 \Omega)^{1 / 2}-O(\log \Omega) .
$$

This result when compared with the formulas (6.3), with which it must be in accord for suitable values of $\Delta$ and $\Omega$, shows that the characteristic values in the present configuration are given by formulas

$$
\begin{aligned}
& S_{n}(\Omega)=-\Omega+(2 n-1)(2 \Omega)^{1 / 2}+O(\log \Omega), \\
& C_{n}(\Omega)=-\Omega+(2 n+1)(2 \Omega)^{1 / 2}+O(\log \Omega) .
\end{aligned}
$$

Finally the computation of the characteristic exponent depends only upon the evaluation of the quantity $\Theta$ given in (25b). This evaluation from the forms (7.6b) and (7.7a) is found in the present case to be

$$
\Theta=\frac{\pi e^{(8 \Omega)^{1 / 2}}}{(32 \Omega)^{\sigma}}\left[\frac{1}{\Gamma\left(\frac{1}{4}-\sigma\right) \Gamma\left(\frac{3}{4}-\sigma\right)}\right]_{1}-1 .
$$

Chapter 8

The CONFIGURATION VIII

8.1. The change of variables. The configuration numbered VIII in Figure 1 is to be defined as that in which $\Delta$ is negative and numerically large, while

$$
-|\Delta|+M_{2}|\Delta|^{1 / 2} \leqq-\Omega \leqq \frac{-1}{M_{1}}|\Delta| .
$$

The substitutions for the transformation of the equation (1) are to be 


$$
\rho=\frac{|\Delta|-\Omega}{|\Delta|^{1 / 2}} e^{\pi i / 2}, \quad \sigma^{2}=1-\frac{\Omega}{|\Delta|}, \quad s=\frac{i}{\sigma}\left(\frac{\pi}{2}-z\right),
$$

in which case the resulting equation of the form (3) has precisely the coefficients (3.3). The value of $\sigma$ is again confined to the range (3.4), and if the half-strip

$$
\begin{aligned}
& R_{z}: \quad 0 \leqq x \leqq \pi \\
& 0 \leqq y
\end{aligned}
$$

is chosen as the domain of $z$, the corresponding region $R_{a}$ is precisely that of (3.6). In terms of $s$, therefore, the present equation coincides entirely with that of chapter 3. The hypotheses are in consequence uniformly fulfilled and Figure 2 again applies. The latter evidently leads in the present instance to Figure 9.
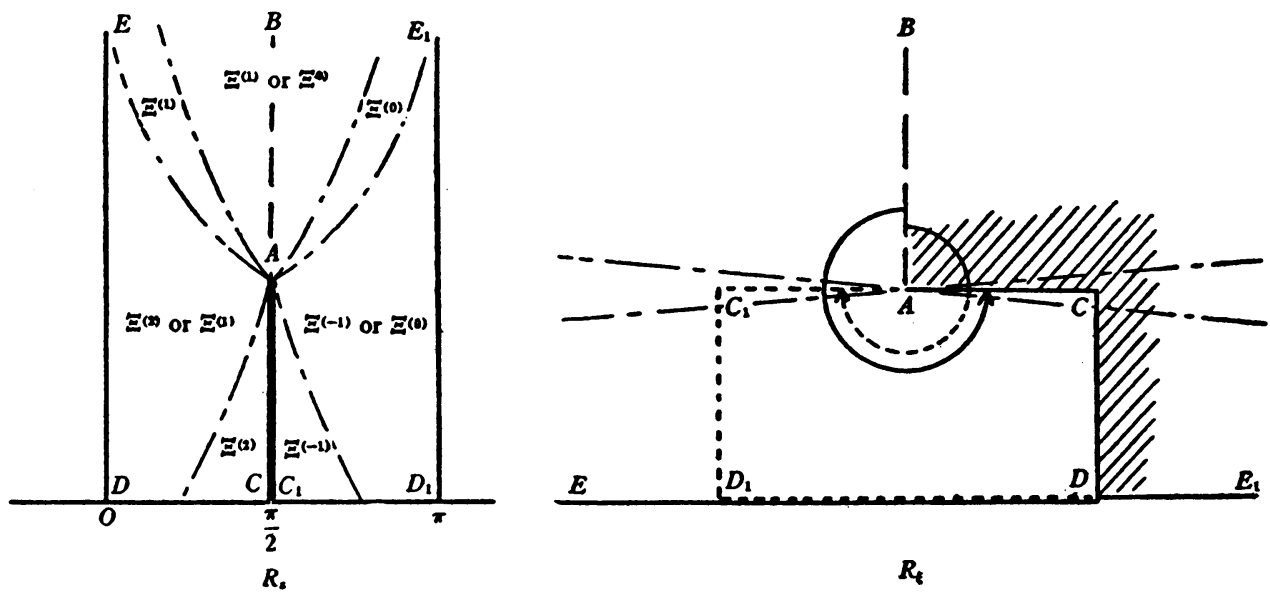

Fig. 9

The extension of the representations which are to be obtained from $R_{z}$ into the entire strip (13) may be made directly by observing that $u_{\alpha}(z)$ and $u_{\beta}(z)$ are respectively odd and even as functions of the variable $(z-\pi / 2)$, and by applying the identities (14a) and (14b) to the formulas for $u_{o}(z)$ and $u_{e}(z)$. With this accomplished the further considerations of $\$ 1.6$ are, of course, applicable.

8.2. The solutions. The zero of $\chi_{0}^{2}$ is of the first order, and, as may be seen from Figure 9, both the points $z=0$ and $z=\pi / 2$ lie in the sub-region $\boldsymbol{\Xi}^{(2)}$. The formulas (11a) and (11b) may, therefore, be drawn upon, with $h=2$, and lead to the formulas which follow. 
When $z$ is in $\Xi^{(l)}$, and $|\xi| \geqq N$,

(8.4a)

$$
u_{o}(z)=\frac{1}{2}\left(\frac{\sigma^{2}}{\rho^{2} \phi_{1} \phi}\right)^{1 / 2}\left\{K_{0,1}^{2, l} e^{i \xi}+K_{0,2}^{2, l} e^{-i \xi}\right\}
$$

(8.5a)

$$
\begin{aligned}
& u_{e}(z)=\frac{1}{2}\left(\frac{\phi_{1}}{\phi}\right)^{1 / 2}\left\{K_{e, 1}^{2, l} e^{i \xi}+K_{e, 2}^{2, l} e^{-i \xi}\right\}, \\
& u_{\alpha}(z)=\frac{1}{2}\left(\frac{\sigma^{2}}{\rho^{2} \phi_{2} \phi}\right)^{1 / 2}\left\{K_{\alpha, 1}^{2, l} e^{i \xi}+K_{\alpha, 2}^{2, l} e^{-i \xi}\right\},
\end{aligned}
$$

\begin{tabular}{|c|c|c|c|c|}
\hline$l$ & -1 & 0 & 1 & 2 \\
\hline$K_{0,1}^{2,2}$ & {$[i] e^{i \xi_{1}}$} & {$[i] e^{i \xi_{1}}$} & {$[1] e^{-i k_{1}}$} & {$[1] e^{-i t_{1}}$} \\
\hline$K_{0,2}^{2,2}$ & {$[-i] e^{-i \xi_{1}}$} & {$[-1] e^{i \xi_{1}}$} & {$[-1] e^{i k_{2}}$} & {$[-1] e^{s f_{1}}$} \\
\hline$K_{0,1}^{, 2}$ & {$[-i] e^{i \xi_{2}}$} & {$[-i] e^{i \xi_{1}}$} & {$[1] e^{-i k_{1}}$} & {$[1] e^{-\lambda \xi_{1}}$} \\
\hline$K_{0,2}^{2,2}$ & {$[-i] e^{i \xi_{1}}$} & {$[1] e^{i \xi_{1}}$} & {$[1] e^{e \xi_{1}}$} & {$[1] e^{e k_{2}}$} \\
\hline
\end{tabular}

$$
u_{\beta}(z)=\frac{1}{2}\left(\frac{\phi_{2}}{\phi}\right)^{1 / 2}\left\{K_{\beta, 1}^{2, l} e^{i \xi}+K_{\beta, 2}^{2, l} e^{-i \xi}\right\},
$$

with coefficients

\begin{tabular}{c|c|c|c|c|}
$l$ & -1 & 0 & 1 & 2 \\
\hline$K_{\alpha, 1}^{2, l}$ & {$[i] e^{i \xi_{2}}$} & {$[i] e^{i \xi_{2}}$} & {$[1] e^{-i \xi_{2}}$} & {$[1] e_{-i \xi_{2}}$} \\
\hline$K_{\alpha, 2}^{2, l}$ & {$[-i] e^{-i \xi_{2}}$} & {$\left[-2 e^{\pi i / 4} \cos \left(\xi_{2}-\frac{\pi}{4}\right)\right]$} & {$\left[-2 e^{\pi i / 4} \cos \left(\xi_{2}-\frac{\pi}{4}\right)\right]$} & {$[-1] e^{\alpha \xi_{2}}$} \\
\hline$K_{\beta, 1}^{2, l}$ & {$[-i] e^{i \xi_{2}}$} & {$[-i] e^{i \xi_{2}}$} & {$[1] e^{-i \xi_{2}}$} & {$[1] e^{-i \xi_{2}}$} \\
\hline$K_{\beta, 2}^{2, l}$ & {$[-i] e^{\alpha \xi_{2}}$} & {$\left[2 e^{-\pi / 4} \cos \left(\xi_{2}+\frac{\pi}{4}\right)\right]$} & {$\left[2 e^{-\pi i / 4} \cos \left(\xi_{2}+\frac{\pi}{4}\right)\right]$} & {$[1] e^{i \xi_{2}}$} \\
\hline
\end{tabular}


When $|\xi| \leqq N$,

$$
u_{0}(z)=\left(\frac{\pi \sigma^{2}}{6 \rho^{2} \phi_{1} \phi}\right)^{1 / 2} \xi^{1 / 6} e^{i \xi_{1}+8 \pi i / 4}\left[\xi^{1 / 8} J_{-1 / 8}(\xi)+\xi^{1 / 8} J_{-1 / 8}(\xi)\right]
$$

$$
\begin{aligned}
& u_{\odot}(z)=\left(\frac{\pi \phi_{1}}{6 \phi}\right)^{1 / 2} \xi^{1 / 6} e^{i \xi_{1}-\pi i / 4}\left[\xi^{1 / 8} J_{-1 / 8}(\xi)+\xi^{1 / 8} J_{1 / 8}(\xi)\right], \\
& u_{\alpha}(z)=-\left(\frac{2 \pi \sigma^{2}}{3 \rho^{2} \phi_{2} \phi}\right)^{1 / 2} \xi^{1 / 6}\left\{e^{\pi i / 6} \sin \left(\xi_{2}+\frac{\pi}{12}\right)\left[\xi^{1 / 8} J_{-1 / 8}(\xi)\right]\right.
\end{aligned}
$$

$$
\begin{aligned}
&\left.+e^{-\pi i / 6} \cos \left(\xi_{2}-\frac{\pi}{12}\right)\left[\xi^{1 / 8} J_{1 / 2}(\xi)\right]\right\}, \\
& u_{\beta}(z)=\left(\frac{2 \pi \phi_{2}}{3 \phi}\right)^{1 / 2} \xi^{1 / 6}\left\{e^{-\pi i / 8} \cos \left(\xi_{2}+\frac{\pi}{12}\right)\left[\xi^{1 / 8} J_{-1 / 8}(\xi)\right]\right. \\
&\left.+e^{\pi i / 3} \sin \left(\xi_{2}-\frac{\pi}{12}\right)\left[\xi^{1 / 8} J_{1 / 8}(\xi)\right]\right\} .
\end{aligned}
$$

For use in these formulas,

$$
\begin{array}{rlrl}
\frac{\rho \phi}{\sigma} & =-\{|\Delta|+\Omega \cos 2 z\}^{1 / 2}, & \\
\frac{\rho \phi_{1}}{\sigma} & =-\{|\Delta|+\Omega\}^{1 / 2}, & \frac{\rho \phi_{2}}{\sigma}=-\{|\Delta|-\Omega\}^{1 / 2}, \\
\xi & =\int_{s_{0}}^{\Omega}\{\Delta-\Omega \cos 2 z\}^{1 / 2} d z, & z_{0}=\frac{1}{2} \cos ^{-1} \Delta / \Omega, \\
\xi_{2} & =\int_{0}^{y_{0}}\{|\Delta|-\Omega \cosh 2 y\}^{1 / 2} d y, & y_{0}=\frac{1}{2} \cosh ^{-1}|\Delta| / \Omega, \\
\xi_{1}=\xi_{2}-i \int_{0}^{\pi / 2}\{|\Delta|+\Omega \cos 2 x\}^{1 / 2} d x . &
\end{array}
$$

It is found that for all real values of $z$ on the interval $(0, \pi)$ the respective formulas are

$$
u_{0}(x)=\frac{[1]}{\{(|\Delta|+\Omega)(|\Delta|+\Omega \cos 2 x)\}^{1 / 4}} \sinh \left[\int_{0}^{x}\{|\Delta|+\Omega \cos 2 x\}^{1 / 2} d x\right],
$$

$$
u_{\bullet}(x)=\left\{\frac{|\Delta|+\Omega}{|\Delta|+\Omega \cos 2 x}\right\}^{1 / 4}[1] \cosh \left[\int_{0}^{x}\{|\Delta|+\Omega \cos 2 x\}^{1 / 2} d x\right] \text {, }
$$




$$
\begin{aligned}
& u_{\alpha}(x)=\frac{[1]}{\{(|\Delta|-\Omega)(|\Delta|+\Omega \cos 2 x)\}^{1 / 4}} \sinh \left[\int_{\pi / 2}^{x}\{|\Delta|+\Omega \cos 2 x\}^{1 / 2} d x\right], \\
& u_{\beta}(x)=\left\{\frac{|\Delta|-\Omega}{|\Delta|+\Omega \cos 2 x}\right\}^{1 / 4}[1] \cosh \left[\int_{x / 2}^{x}\{|\Delta|+\Omega \cos 2 x\}^{1 / 2} d x\right] .
\end{aligned}
$$

The axis of imaginaries in Figure 9 likewise lies in the sub-region $\Xi^{(2)}$, and the formulas for the solutions of the associated Mathieu equation are accordingly found to be

$$
\begin{aligned}
& v_{0}(x)=\frac{[1]}{\{(|\Delta|+\Omega)(|\Delta|+\Omega \cosh 2 x)\}^{1 / 4}} \sin \left[\int_{0}^{x}\{|\Delta|-\Omega \cosh 2 x\}^{1 / 2} d x\right], \\
& v_{\theta}(x)=\left\{\frac{|\Delta|+\Omega}{|\Delta|+\Omega \cosh 2 x}\right\}^{1 / 4}[1] \cos \left[\int_{0}^{x}\{|\Delta|+\Omega \cosh 2 x\}^{1 / 2} d x\right] .
\end{aligned}
$$

8.3. The characteristic exponent. It is evident that the present configuration admits no characteristic values. The formulas (8.4c) and (8.5c) yield the evaluation

$$
\Theta=[2] \cosh ^{2}\left[\int_{0}^{\pi / 2}\{|\Delta|+\Omega \cos 2 x\}^{1 / 2} d x\right]-1,
$$

and the formula (25a) accordingly gives the characteristic exponent in the form

$$
\mu=\left[\frac{2}{\pi} \int_{0}^{\pi / 2}\{|\Delta|+\Omega \cos 2 x\}^{1 / 2} d x\right] .
$$

Clearly, the configuration is one of unstable solutions.

\section{Chapter 9}

\section{THE CONFIGURATION IX}

9.1. The differential equation. In this final configuration to be considered, i.e., IX of Figure 1, the parameter $\Delta$ is large and negative while

$$
-\frac{1}{M_{1}}|\Delta| \leqq-\Omega \leqq 0
$$

The variable is to be restricted to any region in which a relation (2.4a) is fulfilled with some constant $M_{1}$, and this constant is that which figures in (9.1). The substitutions

$$
\rho=|\Delta|^{1 / 2} e^{\pi i / 2}, \quad \sigma^{2}=\frac{\Omega}{|\Delta|}, \quad s=\frac{\pi}{2}-z
$$


reduce the differential equation to the form (3) with the coefficients (2.3), the parameter $\sigma$ being confined as in (2.5). As was remarked in chapter 2 , the Stokes' phenomenon is absent and a single formula serves to describe a solution over the entire strip given by $(2.4 \mathrm{a})$ and $(2.4 \mathrm{~b})$.

9.2. The solutions. The solutions (2.7) apply to the present differential equation (3) and may be used in the formulas (8b). It is found thus that

$$
u_{o}(z)=\frac{i}{2}\left(\frac{1}{\rho^{2} \phi_{1} \phi}\right)^{1 / 2}\left\{e^{i\left(\xi-\xi_{1}\right)}[1]-e^{-i\left(\xi-\xi_{1}\right)}[1]\right\}
$$

$$
\begin{aligned}
& u_{e}(z)=\frac{1}{2}\left(\frac{\phi_{1}}{\phi}\right)^{1 / 2}\left\{e^{i\left(\xi-\xi_{1}\right)}[1]+e^{-i\left(\xi-\xi_{1}\right)}[1]\right\}, \\
& u_{\alpha}(z)=\frac{i}{2}\left(\frac{1}{\rho^{2} \phi_{2} \phi}\right)^{1 / 2}\left\{e^{i \xi}[1]-e^{-i \xi}[1]\right\} \\
& u_{\beta}(z)=\frac{1}{2}\left(\frac{\phi_{2}}{\phi}\right)^{1 / 2}\left\{e^{i \xi}[1]+e^{-i \xi}[1]\right\}
\end{aligned}
$$

with the symbols evaluated by the relations

$$
\begin{gathered}
\rho \phi=i\{|\Delta|+\Omega \cos 2 z\}^{1 / 2}, \\
\rho \phi_{1}=i\{|\Delta|+\Omega\}^{1 / 2}, \quad \rho \phi_{2}=i\{|\Delta|-\Omega\}^{1 / 2}, \\
i \xi=\int_{\pi / 2}^{z}\{|\Delta|+\Omega \cos 2 z\}^{1 / 2} d z, \\
i\left(\xi-\xi_{1}\right)=\int_{0}^{z}\{|\Delta|+\Omega \cos 2 z\}^{1 / 2} d z .
\end{gathered}
$$

For real values of $z$ these formulas are found to reduce precisely to the forms (8.4c) and (8.5c), while the forms which describe the solutions of the equation (2) are again found to be those of (8.6). As in the case of chapter 2 the conclusion is possible that the symbols [] may be dropped from the formulas when $\Omega=0$.

Lastly, the formula for the characteristic exponent is that already given in (8.7), and there are, of course, no characteristic values.

UNIVERSITY OF WISCONSIN, Madison, WIs. 